\title{
CNO ABUNDANCES OF HYDROGEN-DEFICIENT CARBON AND R CORONAE BOREALIS STARS: A VIEW OF THE NUCLEOSYNTHESIS IN A WHITE DWARF MERGER
}

\author{
D. A. García-Hernández ${ }^{1}$, K. H. Hinkle ${ }^{2}$, David. L. Lambert ${ }^{3}$, And K. Eriksson ${ }^{4}$ \\ ${ }^{1}$ Instituto de Astrofísica de Canarias, C/ Via Láctea s/n, 38200 La Laguna, Spain; agarcia@iac.es \\ ${ }^{2}$ National Optical Astronomy Observatory (NOAO), Tucson, AZ 85726, USA; hinkle@ noao.edu \\ ${ }^{3}$ W. J. McDonald Observatory. The University of Texas at Austin. 1 University Station, C1400. Austin, TX 78712-0259, USA; d11@astro.as.utexas.edu \\ ${ }^{4}$ Department of Physics and Astronomy, Uppsala University, Box 515, 75120 Uppsala, Sweden; Kjell.Eriksson@ astro.uu.se \\ Received 2008 December 15; accepted 2009 January 19; published 2009 April 27
}

\begin{abstract}
We present high-resolution $(R \sim 50,000)$ observations of near-IR transitions of $\mathrm{CO}$ and $\mathrm{CN}$ of the five known hydrogen-deficient carbon (HdC) stars and four R Coronae Borealis (RCB) stars. We perform an abundance analysis of these stars by using spectrum synthesis and state-of-the-art MARCS model atmospheres for cool hydrogendeficient stars. Our analysis confirms reports by Clayton and colleagues that those HdC stars exhibiting CO lines in their spectrum and the cool RCB star S Aps are strongly enriched in ${ }^{18} \mathrm{O}$ (with ${ }^{16} \mathrm{O} /{ }^{18} \mathrm{O}$ ratios ranging from 0.3 to 16 ). Nitrogen and carbon are in the form of ${ }^{14} \mathrm{~N}$ and ${ }^{12} \mathrm{C}$, respectively. Elemental abundances for CNO are obtained from $\mathrm{C}$ I, $\mathrm{C}_{2}, \mathrm{CN}$, and $\mathrm{CO}$ lines. Difficulties in deriving the carbon abundance are discussed. Abundances of $\mathrm{Na}$ from $\mathrm{Na} \mathrm{I}$ lines and $\mathrm{S}$ from $\mathrm{S}$ I lines are obtained. Elemental and isotopic CNO abundances suggest that HdC and RCB stars may be related objects, and that they probably formed from a merger of an He white dwarf with a $\mathrm{C}-\mathrm{O}$ white dwarf.
\end{abstract}

Key words: infrared: stars - stars: abundances - stars: atmospheres - stars: chemically peculiar - white dwarfs

Online-only material: color figures

\section{INTRODUCTION}

The category of hydrogen-deficient luminous stars includes, in order of increasing effective temperature, the hydrogendeficient carbon (HdC) stars, the R Coronae Borealis (RCB) stars, and the extreme helium (EHe) stars. A general supposition is that this sequence represents an evolutionary one. Studies of the chemical compositions of stars along the sequence provide one tool for establishing the validity of the claim for an evolutionary sequence and for testing proposed mechanisms for placing stars on the sequence. This study of high-resolution infrared spectra of selected HdC and RCB stars was prompted by the remarkable discovery from medium-resolution infrared spectra that the oxygen in $\mathrm{HdCs}$ was primarily the isotope ${ }^{18} \mathrm{O}$ and not the usual dominant isotope ${ }^{16} \mathrm{O}$ (Clayton et al. 2005, 2007).

The origins of the HdC, RCB, and EHe stars have remained a puzzle for decades. Two different scenarios have survived theoretical and observational scrutiny. In one, the H-deficient supergiant is formed as a consequence of the merger of an He white dwarf (WD) with a carbon-oxygen WD (Webbink 1984; Iben \& Tutukov 1984; Saio \& Jeffery 2002). This is generally referred to as the double-degenerate (DD) scenario. In the other, these H-deficient stars result from a final, postasymptotic giant branch (post-AGB) helium shell flash in the central star of a planetary nebula (PN; Iben et al. 1996). This final flash may transform the PN central star into a cool H-deficient supergiant; the so-called "born-again" scenario, as discussed by, for example, Herwig (2001) and Blöcker (2001). In a version of this scenario, the remaining H-rich envelope is ingested by the He-shell and the ensuing nucleosynthesis includes large-scale conversion of $\mathrm{H}$ to $\mathrm{He}$. This is refered to as the final flash (FF) scenario.

Elemental and isotopic abundances for $\mathrm{C}, \mathrm{N}$, and $\mathrm{O}$ are a powerful tool for discriminating between $\mathrm{HdC}$ and $\mathrm{RCB}$ stars formed by the DD and FF scenarios. Recent studies of the elemental abundances favor the idea that most $\mathrm{HdC}$ and RCB stars have formed via the DD scenario (e.g., Saio \& Jeffery 2002; Rao 2005; Pandey et al. 2006; Clayton et al. 2006). These studies predicted compositions for the DD scenario based on the assumption that the DD scenario did not involve nucleosynthesis during or following accretion of the He white dwarf by the C-O white dwarf. Clayton et al. (2007) suggested with exploratory calculations that the accretion is very rapid and induces nucleosynthesis that converts ${ }^{14} \mathrm{~N}$ by $\alpha$-capture to ${ }^{18} \mathrm{O}$ and results in a high abundance of ${ }^{18} \mathrm{O}$ relative to ${ }^{16} \mathrm{O}$. In contrast, conditions in the FF scenario are unlikely to provide for abundant amounts of ${ }^{18} \mathrm{O}$. Guerrero et al. (2004) report smoothed particle hydrodynamical simulations of merging white dwarfs that predict nucleosynthesis to occur in mergers that might result in an H-deficient single star. Saio \& Jeffery (2002) modeled the merger with a slow accretion rate $\left(10^{-5} M_{\odot} \mathrm{yr}^{-1}\right.$ versus the $150 M_{\odot} \mathrm{yr}^{-1}$ invoked by Clayton et al. 2007), but followed the structure of the merger product during and following accretion as the star evolved from the blue to the red and back to the blue at approximately constant (high) luminosity. Structural changes caused by Heburning and, if the white dwarfs were assumed to have an $\mathrm{H}$ shell, H-burning were followed as were the dredge-ups to the surface. Obviously, nucleosynthesis occurs in a dramatic fashion when the accreting white dwarf exceeds the Chandrasekhar limit.

In this paper, we present and analyze high-resolution spectra in selected intervals in the $K$ band showing $\mathrm{CO}, \mathrm{CN}$, and other lines for a selected sample of HdC and RCB stars. Our goal is to do an abundance analysis and, in particular, to extract the isotopic ratios ${ }^{12} \mathrm{C} /{ }^{13} \mathrm{C},{ }^{16} \mathrm{O} /{ }^{17} \mathrm{O} /{ }^{18} \mathrm{O}$, and ${ }^{14} \mathrm{~N} /{ }^{15} \mathrm{~N}$. Section 2 describes the near-IR observations. The abundance analysis is presented in Section 3. The results of this analysis are discussed in the framework of the DD scenario for the formation of $\mathrm{HdC}$ and RCB stars in Section 4, and concluding remarks are offered in Section 5. 
Table 1

Observation Summary of the Near-IR PHOENIX Observations ${ }^{\mathrm{a}}$

\begin{tabular}{|c|c|c|c|c|}
\hline Star & $\begin{array}{c}\text { Spectral Ranges Observed } \\
\mu \mathrm{m}\end{array}$ & Date & $\begin{array}{c}\text { Spectral Ranges Observed } \\
\mu \mathrm{m}\end{array}$ & Date \\
\hline HD 137613 & $2.332,2.343,2.354,2.366$ & 2006 Jun 18 & $2.251,2.354$ & 2007 Feb 1 \\
\hline HD 175893 & $2.251,2.332,2.343,2.354,2.366$ & 2006 Jun 18 & $\ldots$ & $\ldots$ \\
\hline HD 182040 & $2.251,2.332,2.343,2.354,2.366$ & 2006 Jun 18 & $\ldots$ & $\ldots$ \\
\hline HD 173409 & $2.332,2.343,2.354,2.366$ & 2006 Jun 18 & $\ldots$ & $\ldots$ \\
\hline HD 148839 & $2.332,2.343,2.354,2.366$ & 2006 Jun 18 & $2.251,2.354$ & $2007 \mathrm{Feb} 1$ \\
\hline S Aps & $\ldots$ & $\ldots$ & $2.251,2.354$ & 2007 Feb 1 \\
\hline UW Cen & $\ldots$ & $\ldots$ & $2.251,2.354$ & 2007 Feb 1, 2007 Feb 7 \\
\hline Y Mus & $\ldots$ & $\ldots$ & $2.251,2.354$ & 2007 Feb 1 \\
\hline V854 Cen & $\ldots$ & $\ldots$ & $2.251,2.354$ & $2007 \mathrm{Feb} 1,2007 \mathrm{Feb} 7$ \\
\hline
\end{tabular}

Note. ${ }^{\mathrm{T}}$ The first five objects (with HD names) are the five HdC stars known while the rest of stars are the four RCB stars observed.

\section{OBSERVATIONS AND OVERVIEW OF THE SPECTRA}

Our sample is composed of the five known HdC stars plus four RCB stars. High-resolution infrared spectroscopic observations were carried out on 2006 June 18, 2007 February 1, and 2007 February 7 using the PHOENIX spectrograph at Gemini South (Hinkle et al. 2003). The spectra were obtained with the 0.34 $\operatorname{arcsec}(R=50,000)$ slit at five grating tilts in the $K$-band region each providing a bandpass of about $0.01 \mu \mathrm{m}\left(19.5 \mathrm{~cm}^{-1}\right)$ and centered at 2.251, 2.332, 2.343, 2.354, and $2.366 \mu \mathrm{m}$; the latter four tilts provide essentially complete coverage from $2.327-2.371 \mu \mathrm{m}$. Table 1 lists the spectral regions covered by our PHOENIX observations for each star in our sample. The observed spectra were reduced to intensity as a function of wavelength by using standard tasks in $\mathrm{IRAF}^{5}$ and the telluric features were removed with the help of a spectrum of a hot star observed the same night. The $\mathrm{S} / \mathrm{N}$ of the observed spectra is always $>100$ at the continuum. Wavelengths are given for standard air.

We have constructed the spectral energy distributions (SEDs) of the stars in our sample by using BVRIJHKL photometry available in the literature and the IRAS flux density at $12 \mu \mathrm{m}$, in order to check if our sample stars display a significant infrared excess at $2.3 \mu \mathrm{m}$. In the $K$ band, the flux from $\mathrm{HdC}$ stars is from the photosphere; an infrared excess from circumstellar dust does not contaminate the $K$-band spectra. The photospheric spectrum is also provided for the RCB star S Aps with an effective temperature $T_{\text {eff }}=5400 \mathrm{~K}$ and observed near maximum light. The RCB UW Cen was observed near maximum light but its flux in the $K$ band is predominantly from circumstellar dust so that the photospheric spectrum is greatly obscured or diluted. Even were the star's photosphere observed free of obscuration, the $K$-band spectra would be expected to be free of molecular lines for this star with $T_{\text {eff }}=7400 \mathrm{~K}$. According to magnitude estimates assembled by the American Association of Variable Star Observers (AAVSO), ${ }^{6}$ V854 Cen was observed during a decline of several magnitudes in brightness, suggesting that the circumstellar emission from hot dust probably dominates the observed spectrum. Even at maximum light, V854 Cen has an infrared excess contributing at $2.3 \mu \mathrm{m}$. As in the case of UW Cen, absent an infrared excess, the $K$-band spectrum would not be expected to show molecular lines because $T_{\text {eff }}=6750 \mathrm{~K}$ (Asplund et al. 1998). Lack of $\mathrm{CO}$ and $\mathrm{CN}$ lines in the

\footnotetext{
5 The Image Reduction and Analysis Facility software package (IRAF) is distributed by the National Optical Astronomy Observatories, which is operated by the Association of Universities for Research in Astronomy, Inc., under cooperative agreement with the National Science Foundation.

6 See http://www.aavso.org/.
}

spectrum of several stars is most probably due to their effective temperatures. Tenenbaum et al. (2005) report an absence of CO lines from other RCBs with temperatures $T_{\text {eff }} \geqslant 6500 \mathrm{~K}$. With $T_{\text {eff }}=7250 \mathrm{~K}$ (Asplund et al. 2000), the lack of molecular lines for Y Mus is not surprising.

Before describing the synthesis of the spectra, an overview of the spectral regions is provided here beginning at the shortest wavelengths observed. Figures 1 and 2 show the wavelength interval 2.246-2.256 $\mu \mathrm{m}$. The $\mathrm{CN}$ molecule through its Red System is a contributor across all observed regions but lines of this molecule are especially well seen in this region because it is free of contributions from the first-overtone $\mathrm{CO}$ bands. The most prominent ${ }^{12} \mathrm{C}^{14} \mathrm{~N}$ lines in the synthetic spectra computed for HD 137613 are marked by the broken red lines. The CN lines are prominent in the spectra of HD 137613, HD 175893, HD 182040, and S Aps but also present in HD 148839 and HD 173409 (Figure 3). A few lines of the $\mathrm{C}_{2}$ Phillips bands 0-2 and $1-3$ cross this interval. Among these 0-2 Q34 at $2.2492 \mu \mathrm{m}$, $1-3 \mathrm{Q} 8$ at $2.2497 \mu \mathrm{m}$ and Q10 at $2.2528 \mu \mathrm{m}$ appear either unblended or sufficiently so to be useful in spectrum syntheses of S Aps, HD 137613, HD 175893 and HD 182040. (0-2 R22 at $2.2488 \mu \mathrm{m}$ may also be useful.) The $\mathrm{C}_{2}$ lines are below the detection limit for HD 148839, Y Mus, UW Cen and V854 Cen, while HD 173409 was not observed around $2.251 \mu \mathrm{m}$. (In the available longer wavelength regions, $\mathrm{C}_{2}$ Phillips lines are irretrievably lost among blends with $\mathrm{CN}$ or $\mathrm{CO}$ lines.) The $2.251 \mu \mathrm{m}$ region because of its lower line density is also one in which atomic lines are readily seen. These include a Si I line at $2.2538 \mu \mathrm{m}$, several S I lines at 2.250-2.256 $\mu \mathrm{m}$, and a Fe I line at $2.2473 \mu \mathrm{m}$. Another Fe I line at $2.2493 \mu \mathrm{m}$ is blended with a $\mathrm{CN}$ feature. Lines of Si I, S I, and Fe I are especially prominent in the spectrum of HD 148839 and distinctly broadened in Y Mus and UW Cen (where they are weakly present), and absent from V854 Cen. The atomic lines seen in the RCB stars Y Mus and UW Cen are broader than in other stars in our sample.

Identification of atomic lines in the additional regions observed is a difficult task because of the much higher density of $\mathrm{CO}$ and $\mathrm{CN}$ molecular lines. The $\mathrm{Na}$ I lines at $2.3348 \mu \mathrm{m}$ and $2.3379 \mu \mathrm{m}$ are clearly seen in all $\mathrm{HdC}$ stars. Additional atomic lines are identifiable in the hotter $\mathrm{HdC}$ stars such as $\mathrm{HD}$ $173409(6100 \mathrm{~K})$ and HD $148839(6500 \mathrm{~K})$, and in HD 182040 $(5600 \mathrm{~K})$, as clearly seen in our Figures 3 and 4, where we have ordered the observed spectra (from top to bottom) according to increasing effective temperature. The atomic features are identified as those lines having a similar strength in HD 182040, HD 173409 and HD 148839. We note that HD 173409 (6100 K) and HD 148839 (6500 K) have almost identical spectra. By 


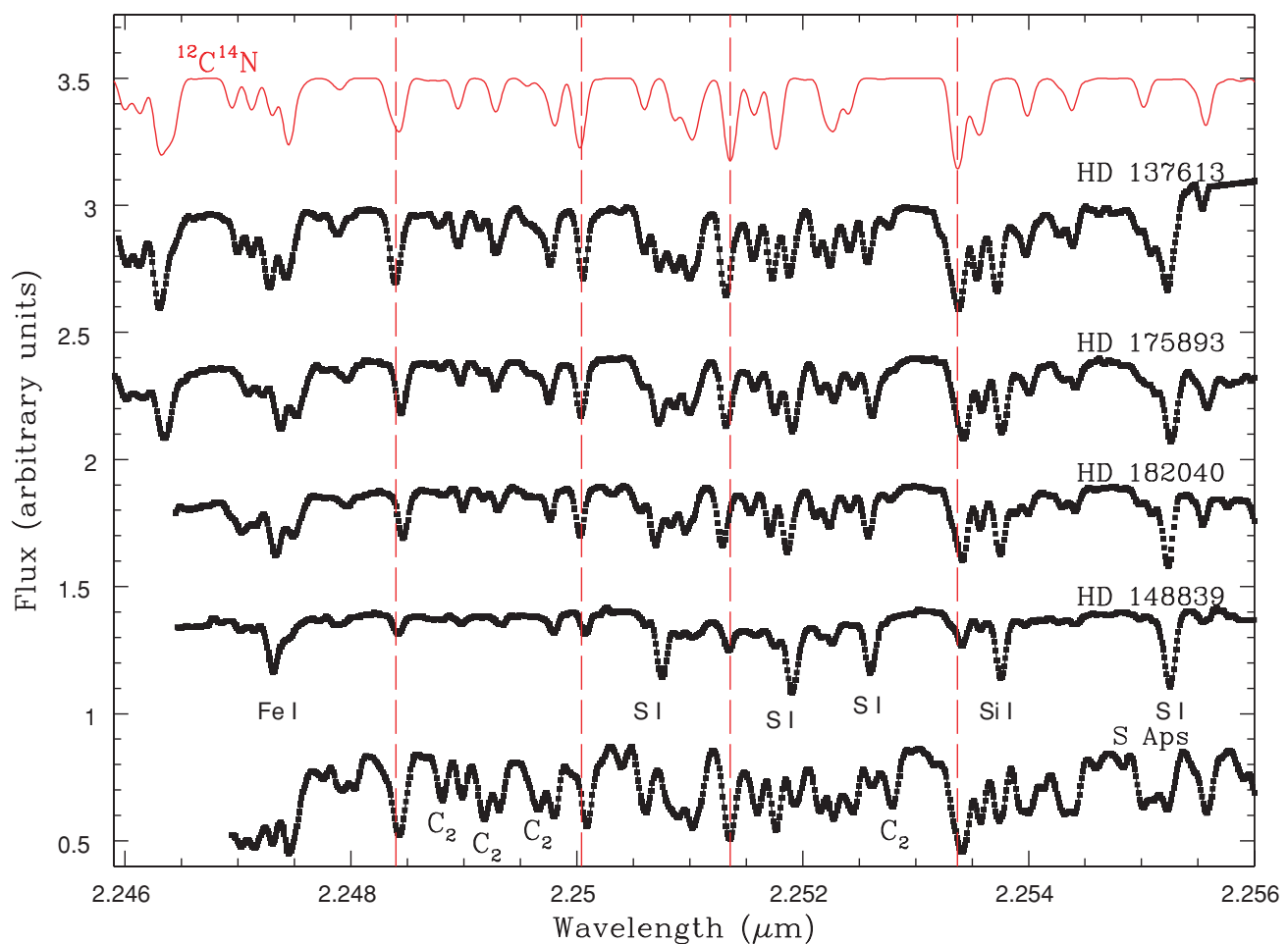

Figure 1. PHOENIX spectra centered at $\sim 2.251 \mu \mathrm{m}$ for four HdC stars (from top to bottom: HD 137613, HD 175893, HD 182040, and HD 148839, respectively). The spectrum of the RCB star S Aps is also shown. Positions of several atomic lines are labeled. The strongest ${ }^{12} \mathrm{C}^{14} \mathrm{~N}$ features are indicated with a dashed red vertical line. $\mathrm{A}^{12} \mathrm{C}^{14} \mathrm{~N}$ synthetic spectrum (in red) composed for HD 137613 is shown at the top. Phillips system $\mathrm{C}_{2}$ lines labeled are 1-3 R22, 0-2 Q34, 1-3 Q8, and 1-3 Q10 in order of increasing wavelength.

(A color version of this figure is available in the online journal.)

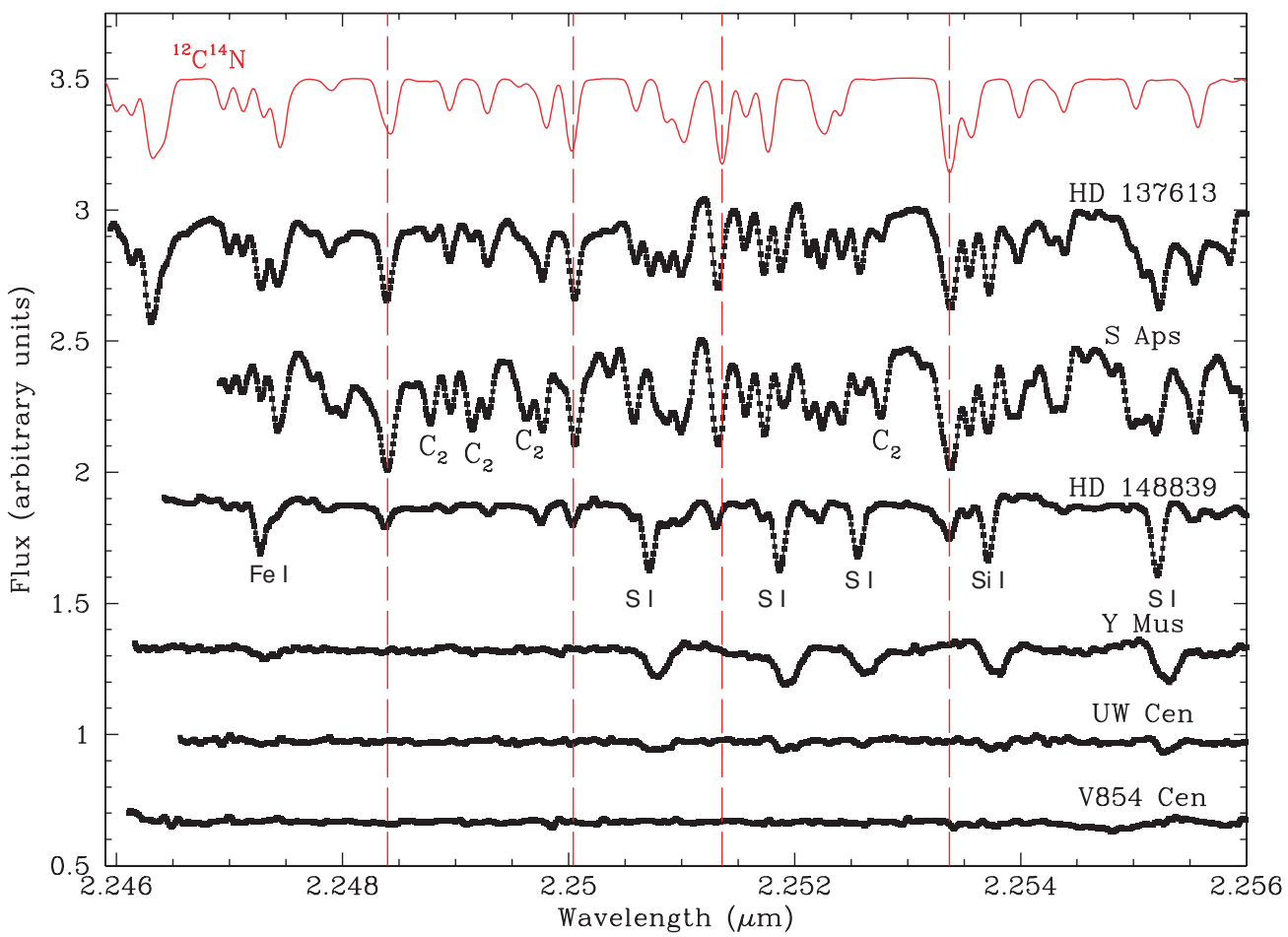

Figure 2. PHOENIX spectra centered at $\sim 2.251 \mu \mathrm{m}$ for four RCB stars and two HdC stars (from top to bottom: HD 137613, S Aps, HD 148839, Y Mus, UW Cen, and V854 Cen, respectively). See the caption of Figure 1 for additional information.

(A color version of this figure is available in the online journal.)

comparing with the infrared spectra of the Sun (Wallace \& Livingston 2003) and of the giant Arcturus (Hinkle et al. 1995), we could identify a few other atomic lines present in the spectra of the warmer HdC stars (see Figures 3, 4, and 5). This is the case 


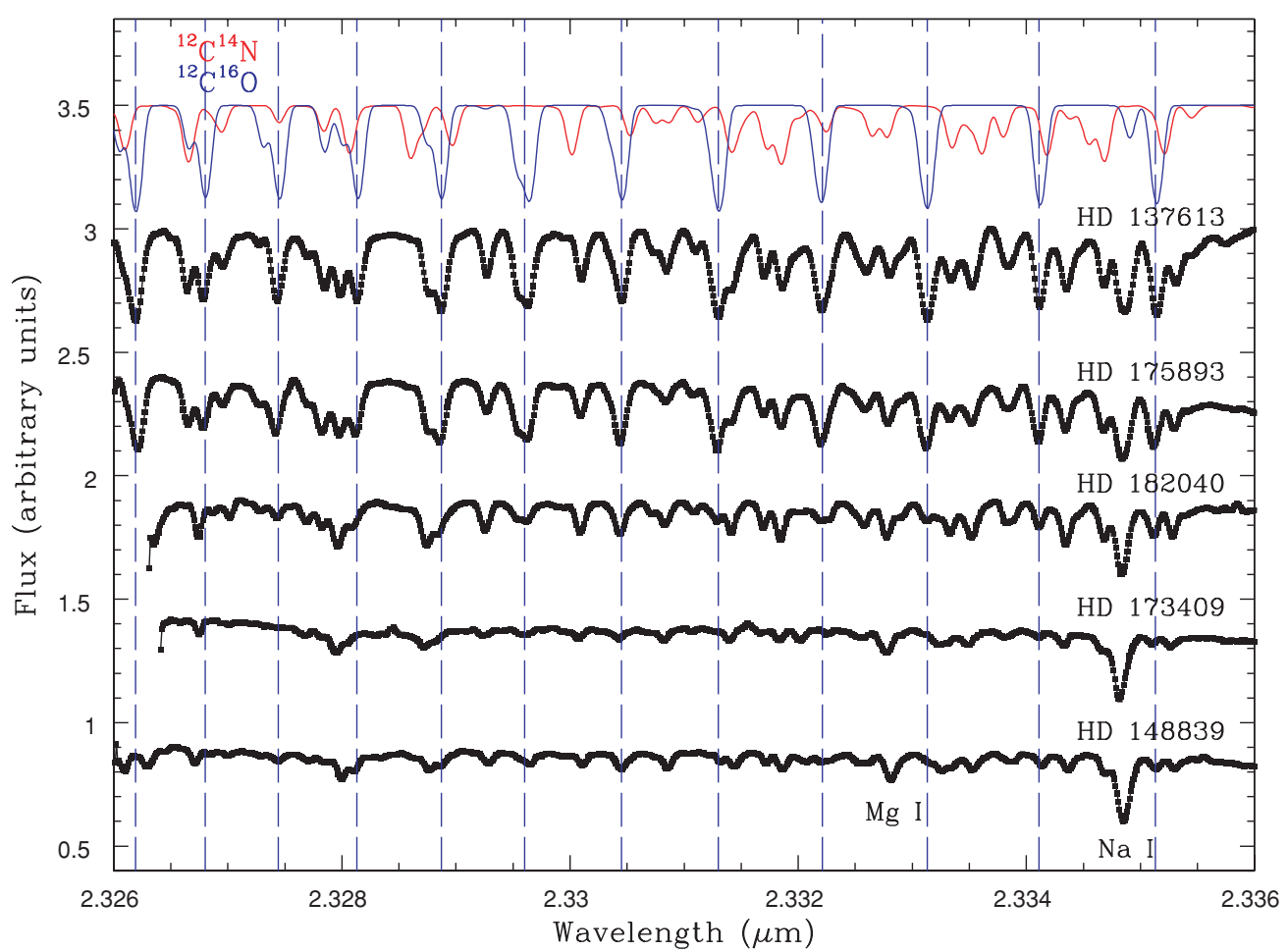

Figure 3. PHOENIX spectra centered at $2.331 \mu \mathrm{m}$ for the five $\mathrm{HdC}$ stars. This region is dominated by lines of the ${ }^{12} \mathrm{C}^{16} \mathrm{O}$ and ${ }^{12} \mathrm{C}^{14} \mathrm{~N}$ molecules but note the Na I line at $2.3348 \mu \mathrm{m}$. The strongest ${ }^{12} \mathrm{C}^{16} \mathrm{O}$ lines are marked with a dashed blue vertical line. Synthetic spectra composed for HD 137613 for the $\mathrm{CO}$ and $\mathrm{CN}$ isomers $\left({ }^{12} \mathrm{C}^{16} \mathrm{O}\right.$ in blue and ${ }^{12} \mathrm{C}^{14} \mathrm{~N}$ in red) shown at the top indicate that these molecules dominate the spectra.

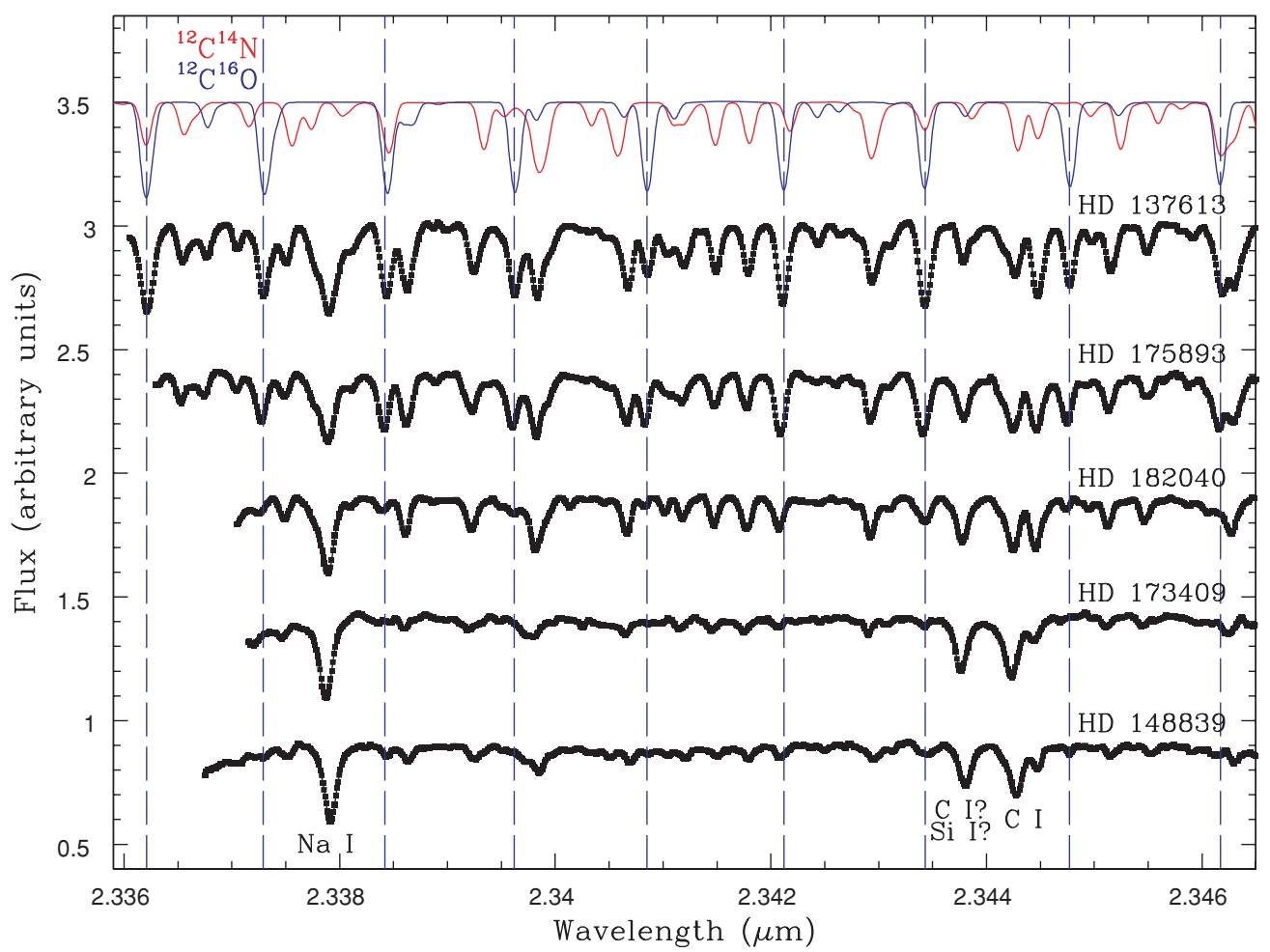

Figure 4. PHOENIX spectra centered at $2.341 \mu \mathrm{m}$ for the five $\mathrm{HdC}$ stars. As for Figure 3, this region is dominated by ${ }^{12} \mathrm{C}^{16} \mathrm{O}$ and ${ }^{12} \mathrm{C}^{14} \mathrm{~N}$ lines but note the $\mathrm{Na}$ I line at $2.3379 \mu \mathrm{m}$. See the caption of Figure 3 for additional information.

for a $\mathrm{Mg}$ I line at $2.3328 \mu \mathrm{m}$, a Fe I line at $2.3566 \mu \mathrm{m}$, and a blend of Si I and Sc I at $2.3579 \mu \mathrm{m}$.

Two other atomic lines are clearly seen at 2.3438 and $2.3443 \mu \mathrm{m}$ (Figure 4). These features are not seen in the Sun and Arcturus. According to the Kurucz and VALD-2 databases, the
$2.3443 \mu \mathrm{m}$ feature is a $\mathrm{C}_{\mathrm{I}}$ line; the line is a close doublet with components at $2.34430 \mu \mathrm{m}$ and $2.34425 \mu \mathrm{m}$. Another (single) $\mathrm{C}$ I line is predicted at $2.3437 \mu \mathrm{m}$, perhaps slightly offset from the atomic feature at $2.3438 \mu \mathrm{m}$ : a difference of about $0.18 \mathrm{~cm}^{-1}$ but the energy levels of upper and lower terms are apparently 


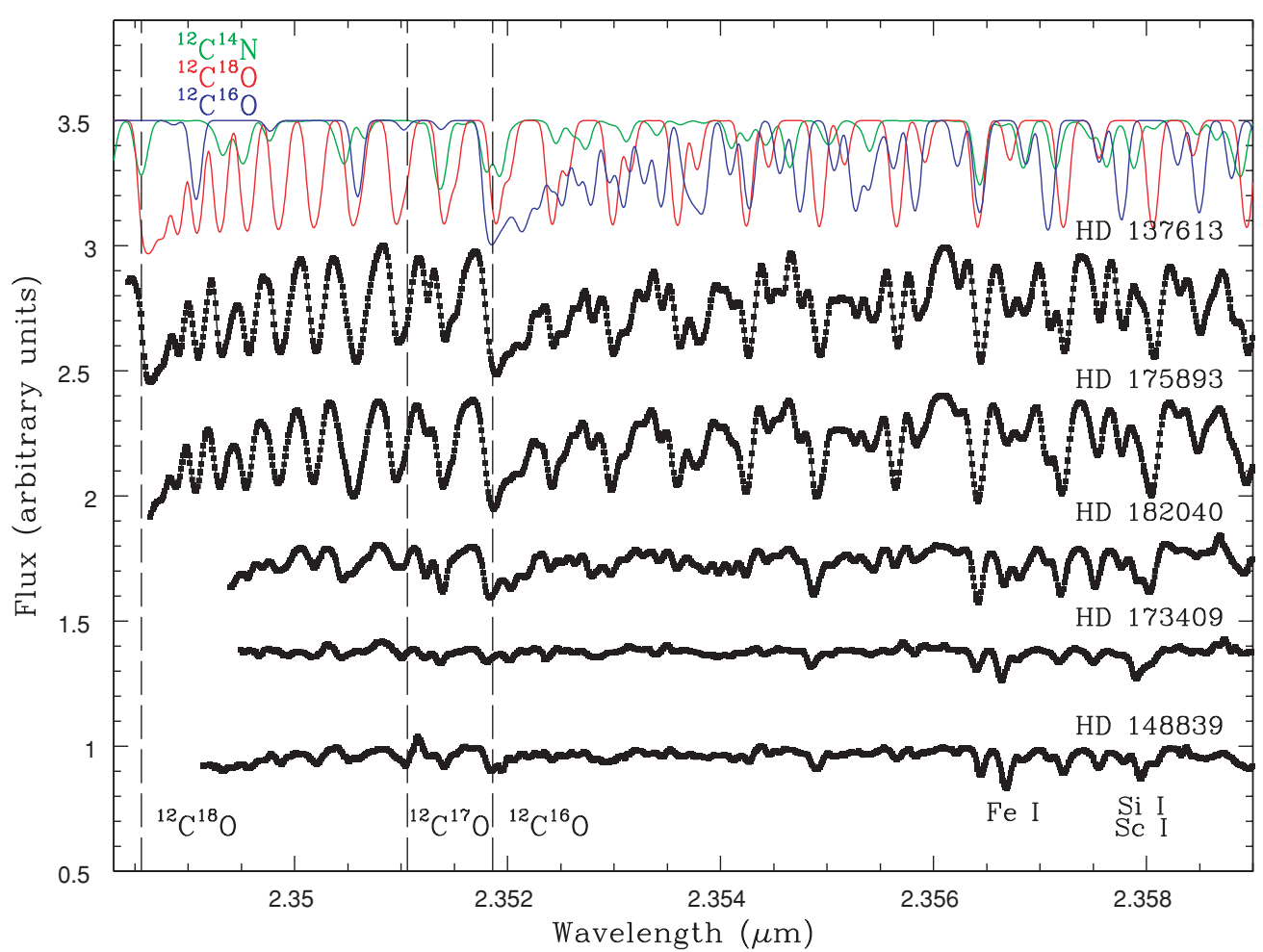

Figure 5. PHOENIX spectra centered at $\sim 2.354 \mu \mathrm{m}$ for the five known HdC stars (from top to bottom: HD 137613, HD 175893, HD 182040, HD 173409, and HD 148839 , respectively). Wavelengths of $2-0{ }^{12} \mathrm{C}^{18} \mathrm{O}, 3-1{ }^{12} \mathrm{C}^{17} \mathrm{O}$, and the $4-2{ }^{12} \mathrm{C}^{16} \mathrm{O}$ bandheads are marked with a vertical dashed line. Synthetic spectra composed for HD 137613 are shown for the isomers ${ }^{12} \mathrm{C}^{16} \mathrm{O}$ in blue, ${ }^{12} \mathrm{C}^{18} \mathrm{O}$ in red and ${ }^{12} \mathrm{C}^{14} \mathrm{~N}$ in green.

known to a higher precision. These C I lines are predictions from the known energy levels (Johansson 1966; Moore 1993). ${ }^{7}$ Wallace \& Hinkle (2007) have recently identified a large number of $\mathrm{C}$ I lines in the near-IR spectrum of the Sun, showing that additional analysis using modern data can significantly extend identification of atomic lines. Unfortunately, they did not identify solar C I lines in our regions. In any case, C I lines are expected to be stronger in our $\mathrm{H}$-deficient and $\mathrm{C}$-rich stars compared to the Sun. Thus, we propose that the feature at $2.3438 \mu \mathrm{m}$ corresponds also to a C I line (although it may be slightly blended with a Si I line). As we will see in Section 3.3, this identification is supported by our spectrum synthesis in the warmer HdC stars.

The 2.326-2.336 $\mu \mathrm{m}$ and 2.336-2.346 $\mu \mathrm{m}$ windows shown in Figures 3 and 4, respectively, provide a selection of $\mathrm{CO}$ lines. The region is crossed by $\mathrm{P}$ and $\mathrm{R}$ lines from the ${ }^{12} \mathrm{C}^{16} \mathrm{O} 2-0$ band with its head at $2.292 \mu \mathrm{m}$ and the 3-1 band with its head at $2.322 \mu \mathrm{m}$; the strong lines are from the $3-1$ band. The $2-0$ bandhead for ${ }^{13} \mathrm{C}^{16} \mathrm{O}$ occurs at $2.344 \mu \mathrm{m}$ but is not an obvious feature in Figure 4 for HD 137613 and HD 175893, the two HdC stars with strong $\mathrm{CO}$ lines. The $2-0$ bandhead for ${ }^{12} \mathrm{C}^{17} \mathrm{O}$ is at $2.3219 \mu \mathrm{m}$ and this isomer is a potential additional contributor to the regions shown in Figures 3 and 4 . The isomer ${ }^{12} \mathrm{C}^{18} \mathrm{O}$ is not a contributor to these regions.

The ${ }^{12} \mathrm{C}^{18} \mathrm{O} 2-0$ band with its head at $2.349 \mu \mathrm{m}$ is captured on Figures 5 and 6 along with the ${ }^{12} \mathrm{C}^{16} \mathrm{O} 4-2$ bandhead. There are also ${ }^{12} \mathrm{C}^{14} \mathrm{~N}$ lines in these regions. The illustrated spectra may also include lines from the $2-0{ }^{13} \mathrm{C}^{16} \mathrm{O}$ band and the 20 and $3-1{ }^{12} \mathrm{C}^{17} \mathrm{O}$ bands (the $3-1$ head is at $2.351 \mu \mathrm{m}$ ). The prominence of ${ }^{12} \mathrm{C}^{18} \mathrm{O}$ lines in the spectrum of the $\mathrm{HdC}$ star

\footnotetext{
7 Curiously, the NIST list of C I energy levels omits the upper levels giving
} the $2.34430 \mu \mathrm{m}$ doublet but includes the upper level giving the $2.3437 \mu \mathrm{m}$ line.
HD 137613 provides the anticipated confirmation of Clayton et al.'s (2005) discovery of a high ${ }^{18} \mathrm{O}$ content in this star from medium resolution spectra. Similarly, our spectra confirm the Clayton et al. (2007) report of the high ${ }^{18} \mathrm{O}$ abundance for HD 175893. Indeed, HD 137613 and HD 175893 are spectroscopic twins. Our spectrum of S Aps (Figure 6) confirms that ${ }^{18} \mathrm{O}$ is present (Clayton et al. 2007) but the isotopic ratio ${ }^{18} \mathrm{O} /{ }^{16} \mathrm{O}$ is by inspection less than in the HdC stars HD 137613 and HD 175893.

The region shown in Figure 7 is longward of the $2-0{ }^{12} \mathrm{C}^{18} \mathrm{O}$ bandhead and, thus, is a mix of ${ }^{12} \mathrm{C}^{16} \mathrm{O},{ }^{12} \mathrm{C}^{18} \mathrm{O}$, and ${ }^{12} \mathrm{C}^{14} \mathrm{~N}$ lines. Synthetic spectra computed for HD 137613 for the CO isomers and for $\mathrm{CN}$ are shown at the top of the figure.

\section{ABUNDANCE ANALYSIS}

\subsection{Synthetic Spectra}

We have performed an abundance analysis by using spectral synthesis techniques and extensive linelists. For this, we have constructed H-deficient MARCS model atmospheres for the spectroscopic effective temperatures (see Table 1) of the stars in our sample. The MARCS models follow the prescription discussed by Asplund et al. (1997). The input chemical composition was taken as representative of the RCB and EHe stars, as determined by Lambert \& Rao (1994), i.e., $\log \epsilon(\mathrm{H})=$ 7.5 and a $\mathrm{C} / \mathrm{He}$ ratio of $1 \%$ by number density. ${ }^{8}$ The input abundances of $\mathrm{O}$ and heavier elements are roughly solar. For the HdC star HD 148839 we have used a less H-deficient model

\footnotetext{
8 The abundances are normalized to $\log \sum \mu_{i} \varepsilon_{i}=12.15$ where $\mu_{i}$ is the mean atomic weight of element $i$, i.e., $\log \varepsilon(\mathrm{He})=11.52$ and $\log \varepsilon(\mathrm{C})=9.52$; see Asplund et al. (1997).
} 


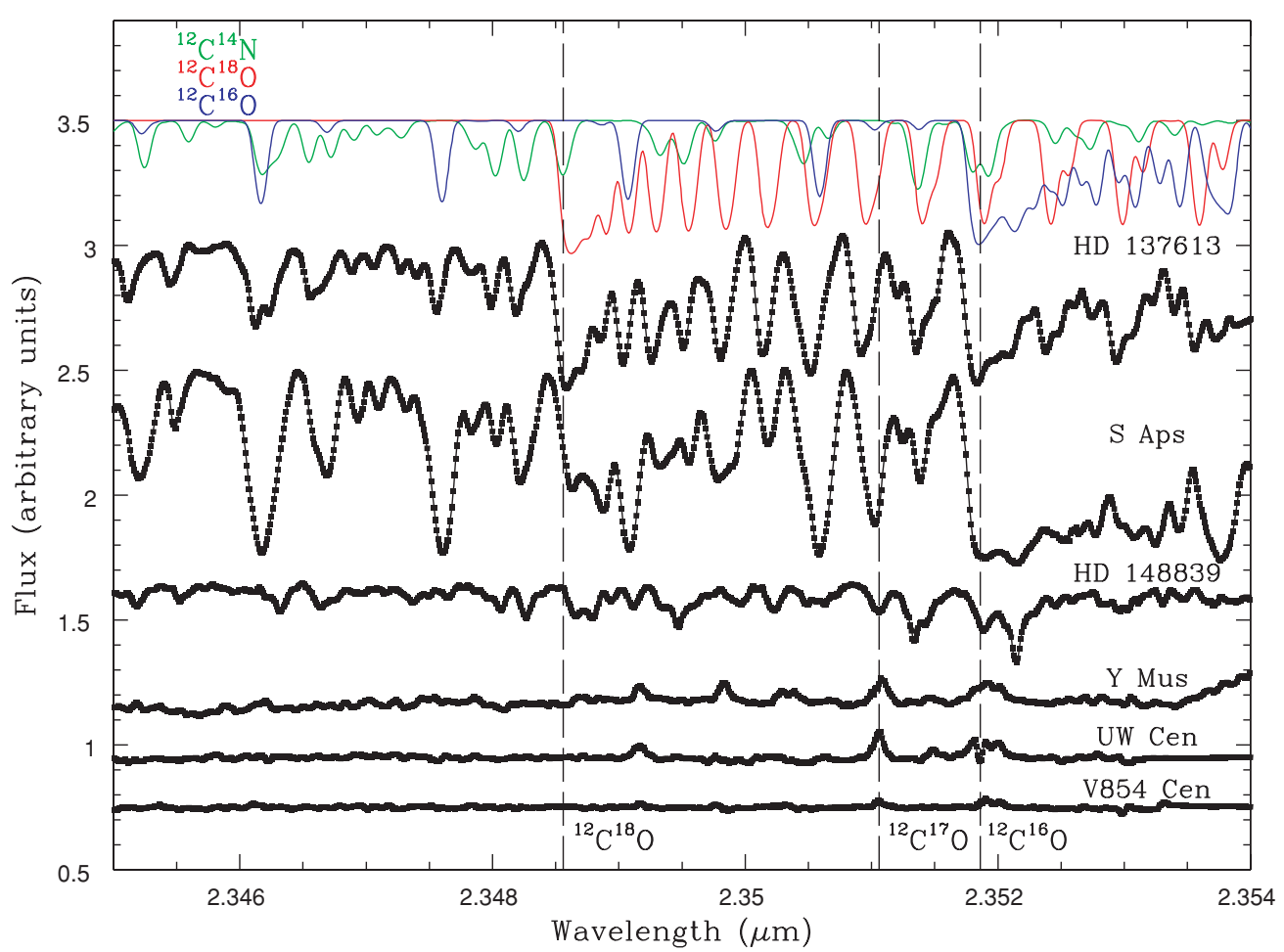

Figure 6. PHOENIX spectra around the $2-0{ }^{12} \mathrm{C}^{18} \mathrm{O}$ at $\sim 2.3485 \mu \mathrm{m}$ for six stars (four RCB stars and two HdC stars): (from top to bottom) HD 137613 , S Aps, HD 148839, Y Mus, UW Cen, and V854 Cen. Synthetic spectra composed for HD 137613 for the CO isomers $\left({ }^{12} \mathrm{C}^{16} \mathrm{O}\right.$ in blue and ${ }^{12} \mathrm{C}^{18} \mathrm{O}$ in red) and for the ${ }^{12} \mathrm{C}^{14} \mathrm{~N}$ isomer (in green) are shown at the top for comparison. The apparent emission features in the spectra of the Y Mus, UW Cen, and V854 Cen arise from incomplete canceling of telluric lines. See the caption of Figure 5 for additional information.

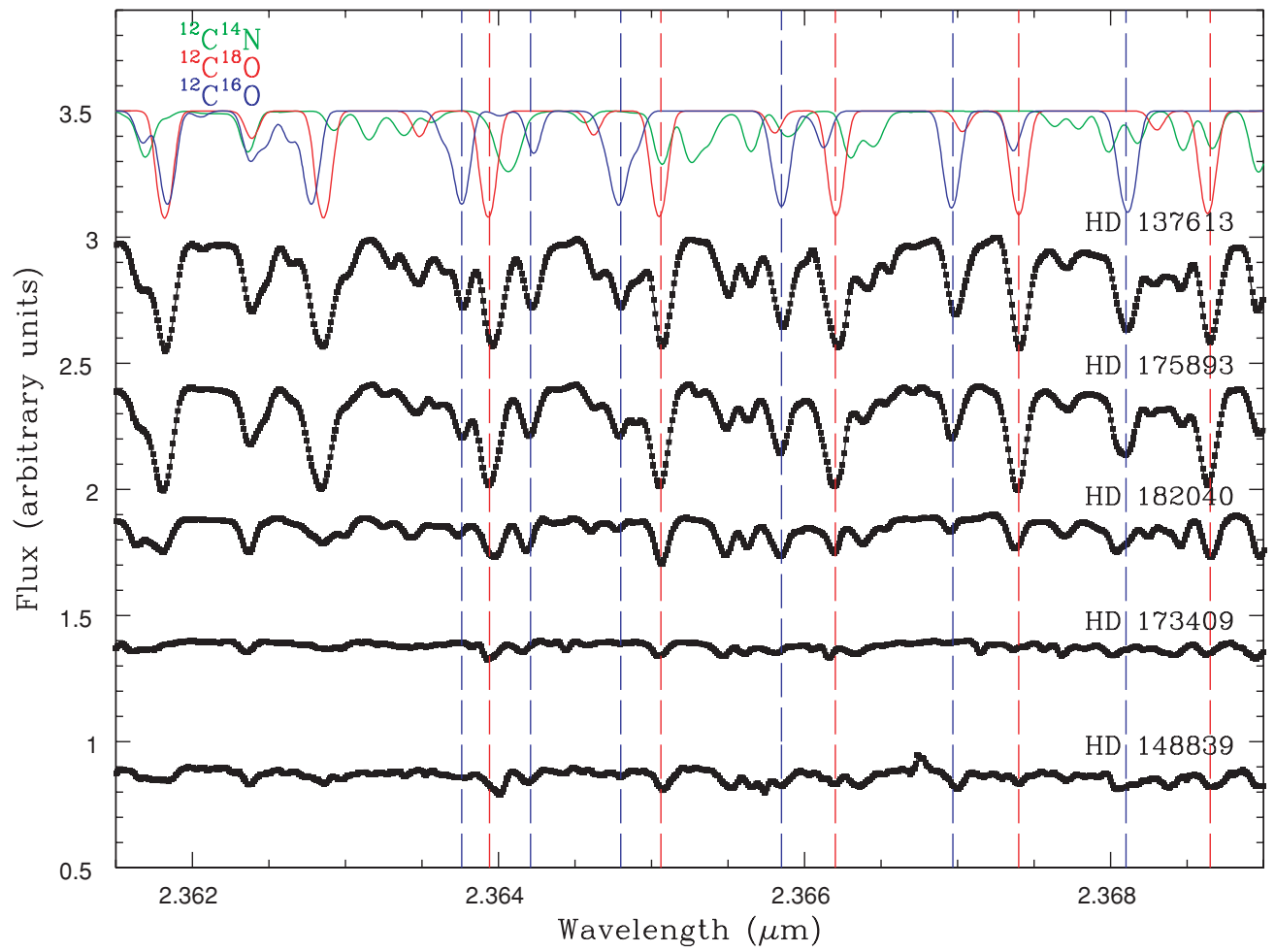

Figure 7. PHOENIX spectra centered at $2.365 \mu \mathrm{m}$ of the five known $\mathrm{HdC}$ stars. Synthetic spectra composed for $\mathrm{HD} 137613$ for the $\mathrm{CO}$ isomers $\left({ }^{12} \mathrm{C}^{16} \mathrm{O}\right.$ in blue and ${ }^{12} \mathrm{C}^{18} \mathrm{O}$ in red) and for the ${ }^{12} \mathrm{C}^{14} \mathrm{~N}$ isomer (in green) are shown at the top for comparison. Note that individual isotopic molecular lines of ${ }^{12} \mathrm{C}^{18} \mathrm{O}$ and ${ }^{12} \mathrm{C}^{16} \mathrm{O}$ are clearly resolved and marked with dashed vertical lines.

with $\log \epsilon(\mathrm{H})=9.82$, as suggested by Warner (1967). The input abundances of "metals" such as $\mathrm{Mg}, \mathrm{Si}, \mathrm{Ca}$, and Fe etc. do not greatly affect our results because they contribute very little opacity and the more abundant $\mathrm{C}$ is the primary electron donor. A small set of models were computed for $\mathrm{C} / \mathrm{He}$ ratios of $0.1 \%$ and $10 \%$. 
Table 2

Derived Chemical Abundances in HdC and RCB Stars ${ }^{\mathrm{a}}$

\begin{tabular}{|c|c|c|c|c|c|c|c|c|c|}
\hline Star & $T_{\text {eff }}(\mathrm{K})^{\mathrm{b}}$ & $\xi\left(\mathrm{km} \mathrm{s}^{-1}\right)$ & ${ }^{16} \mathrm{O} /{ }^{18} \mathrm{O}$ & ${ }^{16} \mathrm{O} /{ }^{17} \mathrm{O}$ & ${ }^{14} \mathrm{~N} /{ }^{15} \mathrm{~N}$ & ${ }^{12} \mathrm{C} /{ }^{13} \mathrm{C}$ & $\mathrm{C} / \mathrm{N} / \mathrm{O}^{\mathrm{c}}$ & $<\mathrm{Na}>$ & $<\mathrm{S}>$ \\
\hline HD 137613 & 5400 & 7 & 0.5 & $>50$ & $>10$ & $>10$ & $9.5 / 9.4 / 8.7$ & 6.9 & 7.6 \\
\hline HD 175893 & 5500 & 7 & 0.3 & $>50$ & $>10$ & $>10$ & $9.5 / 9.2 / 8.7$ & 6.5 & 7.6 \\
\hline HD 182040 & 5600 & 7 & 0.5 & $>8$ & $>10$ & $>10$ & $9.5 / 9.2 / 8.0$ & 6.8 & 7.4 \\
\hline HD 173409 & 6100 & 7 & $\ldots$ & $\ldots$ & $\ldots$ & $\ldots$ & $9.5 / 9.2 / 8.7$ & 7.0 & $\ldots$ \\
\hline HD 148839 & 6500 & 7 & $\ldots$ & $\ldots$ & $\ldots$ & $\ldots$ & 9.5/9.8/9.2 & 7.0 & 7.4 \\
\hline S Aps & 5400 & 7 & 16 & $>80$ & $>10$ & $\ldots$ & 9.5/9.6/9.4 & $\ldots$ & 6.8 \\
\hline Y Mus & 7200 & 10 & $\ldots$ & $\ldots$ & 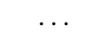 & $\ldots$ & $9.5 / 9.4 / \ldots$ & $\ldots$ & 6.8 \\
\hline $\mathrm{UW} \mathrm{Cen}^{\mathrm{d}}$ & 7400 & 12 & $\ldots$ & $\ldots$ & & $\cdot$ & $9.5 /<9.8 / \ldots$ & $\cdot$ & 6.0 \\
\hline V854 Cen ${ }^{\mathrm{d}}$ & 6750 & 6 & $\cdots$ & $\cdots$ & $\ldots$ & $\ldots$ & $\ldots$ & $\ldots$ & $\ldots$ \\
\hline
\end{tabular}

Notes.

a The abundances are normalized to $\log \sum \mu_{i} \epsilon_{i}=12.15$, i.e., $\log \varepsilon(\mathrm{He})=11.52$ and $\log \varepsilon(\mathrm{C})=9.52$.

${ }^{b}$ References: Clayton et al. (2007); Asplund et al. (1997, 1998); Lawson et al. (1990).

c The CNO abundances are given for the assumed input composition, i.e., $\log \varepsilon(\mathrm{Fe})=7.2, \log \varepsilon(\mathrm{Na})=6.8$, $\log \varepsilon(\mathrm{S})=7.5$, etc. (Asplund et al. 1997).

d The $K$-band spectrum is largely from circumstellar dust and the photospheric spectrum is greatly obscured or diluted.

For $\mathrm{HdC}$ stars, $\mathrm{C} / \mathrm{He}$ is, in principle, measurable from infrared $\mathrm{C}$ I and $\mathrm{C}_{2}$ lines because $\mathrm{He}^{-}$is the dominant opacity source with $\mathrm{C}$ as the leading electron donor. Unfortunately, our spectra provide minimal representation of these lines. A couple of C I lines contribute to our spectra but there are open questions about their identification, especially in the coolest stars, and the accuracy of the $g f$-values. The useful $\mathrm{C}_{2}$ lines are limited to two lines.

For RCB stars, the $\mathrm{C} / \mathrm{He}$ is an ill-determined quantity from observations (Asplund et al. 2000); the predicted equivalent width of an optical $\mathrm{C} \mathrm{I}$ line is insensitive to the $\mathrm{C} / \mathrm{He}$ ratio because the dominant source of continuous opacity is photoionization of neutral carbon atoms from bound levels with an excitation potential similar to that of the $\mathrm{C}_{\mathrm{I}}$ lines. The prediction is verified in part in that the equivalent width of a given $\mathrm{C}_{\mathrm{I}}$ line varies little from star-to-star although the equivalent width of other lines (e.g., Fe I and Fe II lines) may vary considerably. However, analysis of RCB spectra encountered what Gustafsson \& Asplund (1996) called the "carbon problem." The carbon lines are weaker than predicted by the model atmosphere, i.e., "the product of the $g f$-value and carbon abundance provided by the $\mathrm{C}$ I lines is about 0.6 dex less than the product of the $g f$-value provided by quantum chemistry and the abundance assumed in the construction of the model atmosphere." The "carbon problem" for RCB stars is discussed in detail by Asplund et al. (2000). The $\mathrm{C} / \mathrm{He}$ ratio is obtainable for EHe stars for which the carbon problem is not an issue and which provide the mean value of $\mathrm{C} / \mathrm{He}=0.0066$ with a small star-to-star spread (Pandey et al. 2006). By association, this result suggests that a $\mathrm{C} / \mathrm{He}$ of $1 \%$ may be a fair value for RCB and HdC stars.

In addition to the input composition, effective temperature and surface gravity must be chosen. For the $\mathrm{HdC}$ stars (with the exception of HD 148839) and the RCB stars S Aps, UW Cen, and Y Mus, we adopted the $T_{\text {eff }}$ 's estimated from optical spectra and reported by Asplund et al. (2000). For the HdC star HD 148839 we assumed a spectroscopic $T_{\text {eff }}=6500 \mathrm{~K}$ (Lawson et al. 1990). The $T_{\text {eff }}$ of $6750 \mathrm{~K}$ derived from optical spectra (Asplund et al. 1998) was chosen for the RCB star V854 Cen. The chosen $T_{\text {eff }}$ 's for the HdC stars and the RCB star S Aps are those reported by Clayton et al. (2007) for the analysis of their medium resolution near infrared spectra (their Table 2). With regard to the gravities, a surface gravity of $\log g=+0.5$ in cgs units was chosen for the HdC stars and S Aps. The RCB and EHe stars form a sequence in the $\left(T_{\text {eff }}, \log g\right)$ plane with $\log g \simeq+0.5$ expected for the $\mathrm{HdC}$ stars. The surface gravity of the other three RCB stars was also assumed to be $\log g=+0.5$. Asplund et al. (2000) reported $\log g=+0.75$ and +1.0 for $\mathrm{Y}$ Mus and UW Cen, respectively. A microturbulence of $5 \mathrm{~km} \mathrm{~s}^{-1}$ was assumed for the model atmosphere construction. The $T_{\text {eff }}$ adopted for the model atmospheres are listed in Table 2, and, as noted above, all models have $\log g=+0.5$.

Synthetic spectra were generated with the TURBOSPECTRUM package (Alvarez \& Plez 1998) which shares much of its input data and routines with MARCS. In particular, the opacity routines are consistent with those used in the computation of the H-deficient models. The synthetic spectra were calculated for different microturbulent velocities $\left(\xi \sim 3-15 \mathrm{~km} \mathrm{~s}^{-1}\right.$ in steps of $1-2 \mathrm{~km} \mathrm{~s}^{-1}$ ) and were convolved with a Gaussian profile with a width of $6 \mathrm{~km} \mathrm{~s}^{-1}$, which represents the instrumental profile at the resolution of our observations. We obtained an estimate of the microturbulence by comparing synthetic spectra with the observed line widths of the (both weak and strong) ${ }^{12} \mathrm{C}^{16} \mathrm{O}$ lines. For the HdC stars and the RCB star S Aps we found microturbulent velocities of $\sim 7 \mathrm{~km} \mathrm{~s}^{-1}$, a value in good agreement with the mean value of $6.6 \mathrm{~km} \mathrm{~s}^{-1}$ obtained for the cooler RCB stars in their sample by Asplund et al. (2000) and with $\xi=6.8 \mathrm{~km} \mathrm{~s}^{-1}$ for HD 137613 derived by Kipper (2002) from an optical spectrum. For the hotter RCB stars Y Mus and UW Cen, which do not show detectable $\mathrm{CO}$ lines, the microturbulent velocities of 10 and $12 \mathrm{~km} \mathrm{~s}^{-1}$ (Asplund et al. 2000), respectively, were adopted. These higher microturbulences are consistent with the broader appearance of the atomic lines in the $2.251 \mu \mathrm{m}$ region. In addition, we need to convolve the synthetic spectra for these two RCB stars with a larger Gaussian profile $\left(\sim 20-25 \mathrm{~km} \mathrm{~s}^{-1}\right)$ in order to take into account a higher macroturbulence. We will adopt a maximum error of $\pm 2 \mathrm{~km} \mathrm{~s}^{-1}$ for the microturbulence in computing our derived chemical abundances (see Table 3 ).

Synthetic spectra were computed using a list of atomic and molecular lines. Key contributors of lines are the $\mathrm{CO}, \mathrm{CN}$, and $\mathrm{C}_{2}$ molecules. Line positions for ${ }^{12} \mathrm{C}^{16} \mathrm{O},{ }^{12} \mathrm{C}^{17} \mathrm{O},{ }^{12} \mathrm{C}^{18} \mathrm{O}$, and ${ }^{13} \mathrm{C}^{16} \mathrm{O}$ were computed from molecular constants for ${ }^{12} \mathrm{C}^{16} \mathrm{O}$ (Huber \& Herzberg 1979) with constants for isomers computed via standard relations. Oscillator strengths were taken from Goorvitch (1994). The CO dissociation energy was put at $\mathrm{D}_{0}(\mathrm{CO})=11.09 \mathrm{eV}$ for all isomers. A line list for ${ }^{12} \mathrm{C}^{14} \mathrm{~N}$ and its isomers was kindly provided by B. Plez (2007, private communication; see Hill et al. 2002 for a discussion of Plez's linelist). The dissociation was put at $\mathrm{D}_{0}(\mathrm{CN})=7.76 \mathrm{eV}$ (Costes 
Table 3

Sensitivity of the Derived ${ }^{16} \mathrm{O} /{ }^{18} \mathrm{O}$ Ratio and N, O, Na and S Abundances to Slight Changes in the Model Atmosphere Parameters for the HdC star HD 175893

\begin{tabular}{lcccccc}
\hline \hline Adopted Value & Change & $\Delta{ }^{16} \mathrm{O} /{ }^{18} \mathrm{O}$ & $\Delta \log \epsilon(\mathrm{N})$ & $\Delta \log \epsilon(\mathrm{O})$ & $\Delta \log \epsilon(\mathrm{Na})$ & $\Delta \log \epsilon(\mathrm{S})$ \\
\hline$T_{\text {eff }}=5500 \mathrm{~K}$ & $\Delta T_{\text {eff }}= \pm 100 \mathrm{~K}$ & $\mp 0.1$ & \pm 0.1 & \pm 0.1 & \pm 0.05 & \pm 0.05 \\
$\log g=+0.5$ & $\Delta \log g= \pm 1.0$ & $\mp 0.1$ & $-0.2 /+0.4$ & $-0.1 /+0.2$ & $-0.2 /+0.7$ & $-0.2 /+0.4$ \\
$\xi=7 \mathrm{~km} \mathrm{~s}^{-1}$ & $\Delta \xi= \pm 2 \mathrm{~km} \mathrm{~s}^{-1}$ & \pm 0.2 & \pm 0.05 & \pm 0.05 & \pm 0.1 & \pm 0.1 \\
$F W H M=6 \mathrm{~km} \mathrm{~s}^{-1}$ & $\Delta F W H M= \pm 2 \mathrm{~km} \mathrm{~s}^{-1}$ & $\mp 0.05$ & \pm 0.05 & \pm 0.05 & \pm 0.1 & \pm 0.1 \\
\hline
\end{tabular}

et al. 1990). Lines of the $\Delta v=-2$ sequence of the $\mathrm{C}_{2}$ Phillips system cross observed spectral windows and, as noted in Section 2, two or three are present unblended or almost so in the spectra of several of our stars. Line positions are taken from Davis et al. (1988) and oscillator strengths calculated from band oscillator strengths (Kokkin et al. 2007) and Hönl-London factors (Lambert et al. 1986). The empirical dissociation energy of $D_{0}=6.297 \mathrm{eV}$ measured by Urdahl et al. (1991) is used.

For atomic lines, the primary source of information was the VALD-2 database (Kupka et al. 1999). As noted above, identification of atomic features was made using the infrared atlas $(R \sim 100,000)$ of the giant Arcturus (Hinkle et al. 1995) and the infrared solar spectrum (Wallace \& Livingston 2003). The $g f$-value of an atomic lines was adjusted so that the predicted line strength matched the observed strength in the Arcturus atlas. For this, a MARCS model atmosphere was used with Arcturus's fundamental parameters determined by Decin et al. (2003): $T_{\text {eff }}=4300 \mathrm{~K}, \log g=1.50,[\mathrm{M} / \mathrm{H}]=-0.5, \xi=$ $1.7 \mathrm{~km} \mathrm{~s}^{-1}, \mathrm{C} / \mathrm{N} / \mathrm{O}=7.96 / 7.55 / 8.67$, and ${ }^{12} \mathrm{C} /{ }^{13} \mathrm{C}=7$. These parameters are in good agreement with other determinations reported in the literature. Finally, we also test that our molecular linelists reproduce well the observed high-resolution near-IR spectrum of Arcturus. The agreement is especially good for $\mathrm{CO}$ where the $g f$-values and wavelengths are well known. The agreement is also good for the few $\mathrm{CN}$ features around $2.251 \mu \mathrm{m}$ observed in the Arcturus's spectrum and this region will be used for our $\mathrm{N}$ abundance determination. We note that our $\mathrm{CN}$ linelist contains a few incorrect wavelengths but, in general, affected lines are easily identified from a comparison of synthetic and observed spectra. In addition, when stellar $\mathrm{CN}$ is strong there could be more stellar $\mathrm{CN}$ lines than those present in our linelists (see Section 3.2.3). This is the case of the 4-6 CN Red System band which has recently been shown to have a lot of lines in our regions (Davis et al. 2005). Unfortunately, the $g f$-values of these $\mathrm{CN}$ lines are unknown. The $\mathrm{C}_{2}$ lines are absent, as expected, from the Arcturus spectrum.

Synthetic spectra were computed and matched to the observed spectra. Our primary goal was to determine the isotopic ratios for $\mathrm{C}, \mathrm{N}$, and $\mathrm{O}$, as the determination of these ratios is almost independent of the adopted model parameters. The secondary goals were to constrain the $\mathrm{C}, \mathrm{N}$, and $\mathrm{O}$ elemental abundances and to determine the abundances of heavier elements, principally $\mathrm{Na}$ and $\mathrm{S}$.

\subsection{The Isotopic Ratios for $C, N$, and $O$}

For the HdCs HD 137613, HD 175893, and the RCB S Aps, we are able to obtain reliable estimates of the isotopic ratio ${ }^{16} \mathrm{O} /{ }^{18} \mathrm{O}$. For the HdC star HD 182040 , we estimate the ${ }^{16} \mathrm{O} /{ }^{18} \mathrm{O}$ ratio from a few weak features. For HdC stars HD 148839 and HD 173409 and the other RCBs, identification of CO in any isotopic combination is uncertain so that we are decline to give isotopic ratios. For three $\mathrm{HdCs}$ and $\mathrm{S} A \mathrm{Aps}$, lower limits are determined for the ratios ${ }^{12} \mathrm{C} /{ }^{13} \mathrm{C},{ }^{14} \mathrm{~N} /{ }^{15} \mathrm{~N}$, and ${ }^{16} \mathrm{O} /{ }^{17} \mathrm{O}$.

\subsubsection{HD 175893}

In Figures 8-12, we illustrate the fit of synthetic spectra to the observed spectra for HD 175893. The 2.246-2.256 $\mu \mathrm{m}$ region (Figure 8), as noted above, is free of first-overtone CO lines and rich in CN Red System lines. The computed spectrum (red line) with $\log \epsilon\left({ }^{14} \mathrm{~N}\right)=9.2$, the input ${ }^{12} \mathrm{C}$ abundance $(=9.52)$, and the total $\mathrm{O}$ abundance $(=8.7)$ from the isotopes ${ }^{16} \mathrm{O}$ and ${ }^{18} \mathrm{O}$ is a good fit to the observed spectrum. A few lines in the observed spectrum are absent from the adopted linelist. For a few $\mathrm{CN}$ lines, a small revision of their adopted wavelengths is indicated but not here applied. The synthetic spectrum (blue line) corresponding to a reduction of the $\mathrm{N}$ abundance to 8.7 with adopted $\mathrm{C}$ and $\mathrm{O}$ abundances left unchanged reveals which lines arise wholly or primarily from ${ }^{12} \mathrm{C}^{14} \mathrm{~N}$. The fact that the majority of the line depths of many lines vary approximately in the ratio of the change of the $\mathrm{N}$ abundance shows that the lines are not severely saturated. The $\mathrm{N}$ abundance derived from $\mathrm{CN}$ lines is sensitive to the adopted $\mathrm{C}$ and $\mathrm{O}$ abundance. The $\mathrm{CN}$ number density in the atmosphere depends on the product of the partial pressures of $\mathrm{C}$ and $\mathrm{N}$. The partial pressure of $\mathrm{C}$ is determined by both the $\mathrm{C}$ and $\mathrm{O}$ abundances through the formation of $\mathrm{CO}$. The partial pressure of $\mathrm{N}$ is determined by the abundance of $\mathrm{N}$ with minor depletion of free nitrogen from $\mathrm{N}_{2}$ formation and is insensitive to the $\mathrm{C}$ and $\mathrm{O}$ abundances. This region is used to set the limit ${ }^{14} \mathrm{~N} /{ }^{15} \mathrm{~N}>10$. This lax limit on ${ }^{15} \mathrm{~N}$ arises because the strongest $\mathrm{CN}$ lines are just 30 per cent deep and the line density of ${ }^{12} \mathrm{C}^{14} \mathrm{~N}$ lines is high.

The ${ }^{16} \mathrm{O}$ abundance is determined from the $2.326-2.336 \mu \mathrm{m}$ and 2.336-2.346 $\mu \mathrm{m}$ regions shortward of the ${ }^{12} \mathrm{C}^{18} \mathrm{O} 2-0$ bandhead. Synthetic and observed spectra for these regions for HD 175893 are shown in Figures 9 and 10. The syntheses adopt the ${ }^{14} \mathrm{~N}$ abundance $(=9.2)$ from the fit to the $\mathrm{CN}$ lines in Figure 8, and the input $\mathrm{C}$ abundance $(=9.52)$. Syntheses are shown for the same total $\mathrm{O}$ abundance (the sum of ${ }^{16} \mathrm{O}$ and ${ }^{18} \mathrm{O}$ abundances) but two choices of isotopic ratio and, hence, two different ${ }^{16} \mathrm{O}$ abundances. The ${ }^{12} \mathrm{C}^{16} \mathrm{O}$ lines are well fit for an abundance $\log \epsilon\left({ }^{16} \mathrm{O}\right)=8.1$ (i.e., corresponding to the isotopic ratio ${ }^{16} \mathrm{O} /{ }^{18} \mathrm{O}=0.3$ and total $\mathrm{O}$ abundance of $\log \varepsilon(\mathrm{O})=8.7$, see below). The ${ }^{13} \mathrm{C}^{16} \mathrm{O} 2-0$ bandhead at $2.3441 \mu \mathrm{m}$ is just captured on Figure 10. A limit ${ }^{12} \mathrm{C} /{ }^{13} \mathrm{C}>10$ is set using synthetic spectra with different abundances of ${ }^{13} \mathrm{C}$.

The ${ }^{12} \mathrm{C}^{18} \mathrm{O} 2-0$ band is captured on Figures 11 and 12. The synthetic spectra give us a ${ }^{16} \mathrm{O} /{ }^{18} \mathrm{O}$ ratio of 0.3 : the total $\mathrm{O}$ $\left({ }^{16} \mathrm{O}+{ }^{18} \mathrm{O}\right)$ abundance is $\log \varepsilon(\mathrm{O})=8.7$ with $\log \epsilon\left({ }^{16} \mathrm{O}\right)=$ 8.1 and $\log \epsilon\left({ }^{18} \mathrm{O}\right)=8.6$. The best synthetic spectra displayed in these figures are computed for the input $\mathrm{C}$ abundance and the $\mathrm{CN}$ lines are included with the $\mathrm{N}$ abundance derived from Figure 8 . The sensitivity of the derived ${ }^{16} \mathrm{O} /{ }^{18} \mathrm{O}$ ratio to the adopted atmospheric parameters is summarized in Table 3. Our measurement of this isotopic ratio is in good agreement with the Clayton et al. (2007) estimate of ${ }^{16} \mathrm{O} /{ }^{18} \mathrm{O}=0.2$ from the intensities of the two bandheads, as measured off medium resolution spectra. 


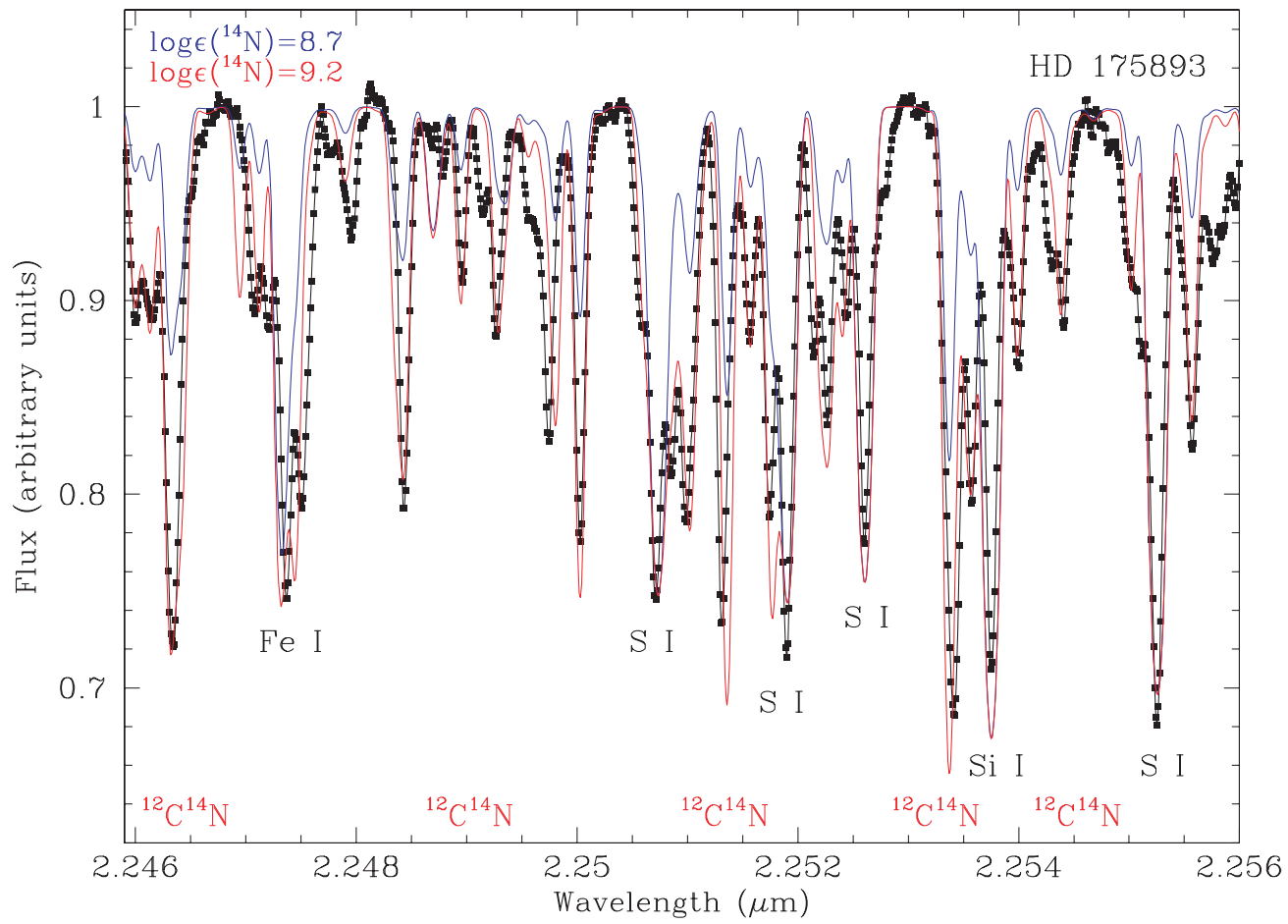

Figure 8. Best synthetic (red) and observed (black) spectrum for the $\mathrm{CN}$ region centered at $\sim 2.251 \mu \mathrm{m}$ for the ${ }^{18} \mathrm{O}-$ rich $\mathrm{HdC}$ star $\mathrm{HD} 175893$. The synthetic spectrum has been created assuming the input $\mathrm{C}$ abundance $\log \varepsilon(\mathrm{C})=9.52$, the $\mathrm{N}$ is in the form of ${ }^{14} \mathrm{~N}$ with an abundance $\log \varepsilon(\mathrm{N})=9.2$, and a total $\mathrm{O}$ abundance of $\log \varepsilon(\mathrm{O})$ $=8.7$. A synthetic spectrum (blue) for a lower ${ }^{14} \mathrm{~N}$ abundance of $\log \varepsilon(\mathrm{N})=8.7$ dex is shown for comparison. Note the strong dependence of the ${ }^{12} \mathrm{C}^{14} \mathrm{~N}$ lines to changes of the elemental $\mathrm{N}$ abundance.

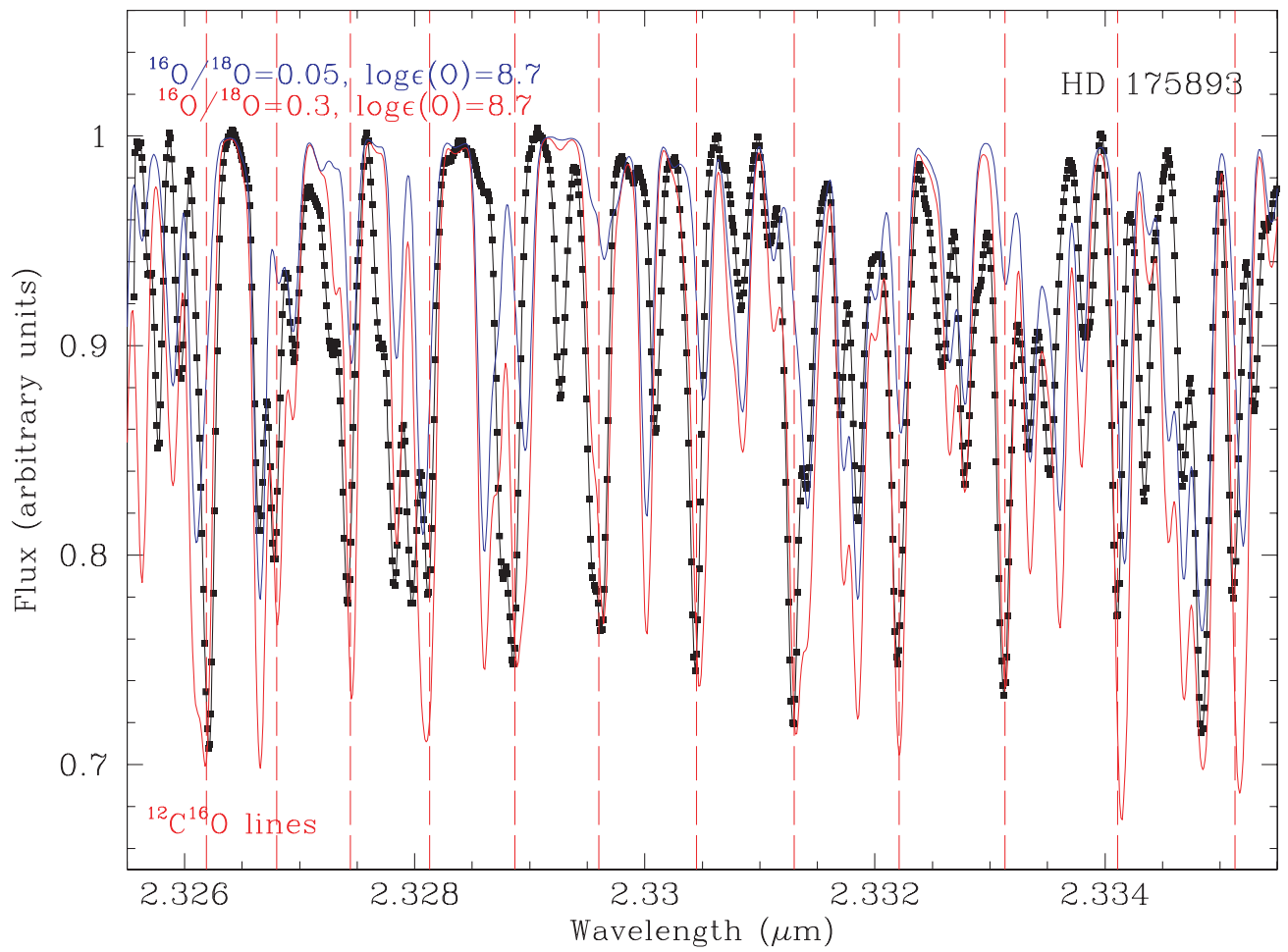

Figure 9. Best synthetic (red) and observed (black) spectrum for the wavelength region centered around $2.332 \mu \mathrm{m}$ for the ${ }^{18} \mathrm{O}-\mathrm{rich} \mathrm{HdC}$ star $\mathrm{HD} 175893$. The red spectrum assumes the input $\mathrm{C}$ abundance of 9.52 , the $\mathrm{N}$ abundance of 9.2 , a total $\mathrm{O}\left({ }^{16} \mathrm{O}+{ }^{18} \mathrm{O}\right)$ of $\log \varepsilon(\mathrm{O})=8.7$, and the isotopic ratio ${ }^{16} \mathrm{O} /{ }^{18} \mathrm{O}=0.3$. This region contains ${ }^{12} \mathrm{C}^{16} \mathrm{O}$ but not ${ }^{12} \mathrm{C}^{18} \mathrm{O}$ lines and the ${ }^{12} \mathrm{C}^{16} \mathrm{O}$ lines are fit with the isotopic ratio ${ }^{16} \mathrm{O} /{ }^{18} \mathrm{O}=0.3\left(\right.$ or $\log \varepsilon\left({ }^{16} \mathrm{O}\right)=8.1$ ). The synthetic spectrum also includes $\mathrm{CN}$ lines. A synthetic spectrum (blue) for a lower ${ }^{16} \mathrm{O} /{ }^{18} \mathrm{O}$ ratio of 0.05 (or a lower ${ }^{16} \mathrm{O}$ abundance) but the same total $\mathrm{O}\left({ }^{16} \mathrm{O}+{ }^{18} \mathrm{O}\right)$ of $\log \varepsilon(\mathrm{O})=8.7$ is shown for comparison. Note the strong dependence of the most clear ${ }^{12} \mathrm{C}^{16} \mathrm{O}$ lines (marked with red dashed vertical lines) to changes of the ${ }^{16} \mathrm{O}$ abundance.

Our estimate of the ${ }^{17} \mathrm{O}$ abundance is obtained from the ${ }^{12} \mathrm{C}^{17} \mathrm{O} 3-1$ band with its head at $2.3511 \mu \mathrm{m}$ and a few $2-0$ and
$3-1{ }^{12} \mathrm{C}^{17} \mathrm{O}$ lines longward of the head. By spectrum synthesis (Figure 11), we find no convincing evidence for the presence of 


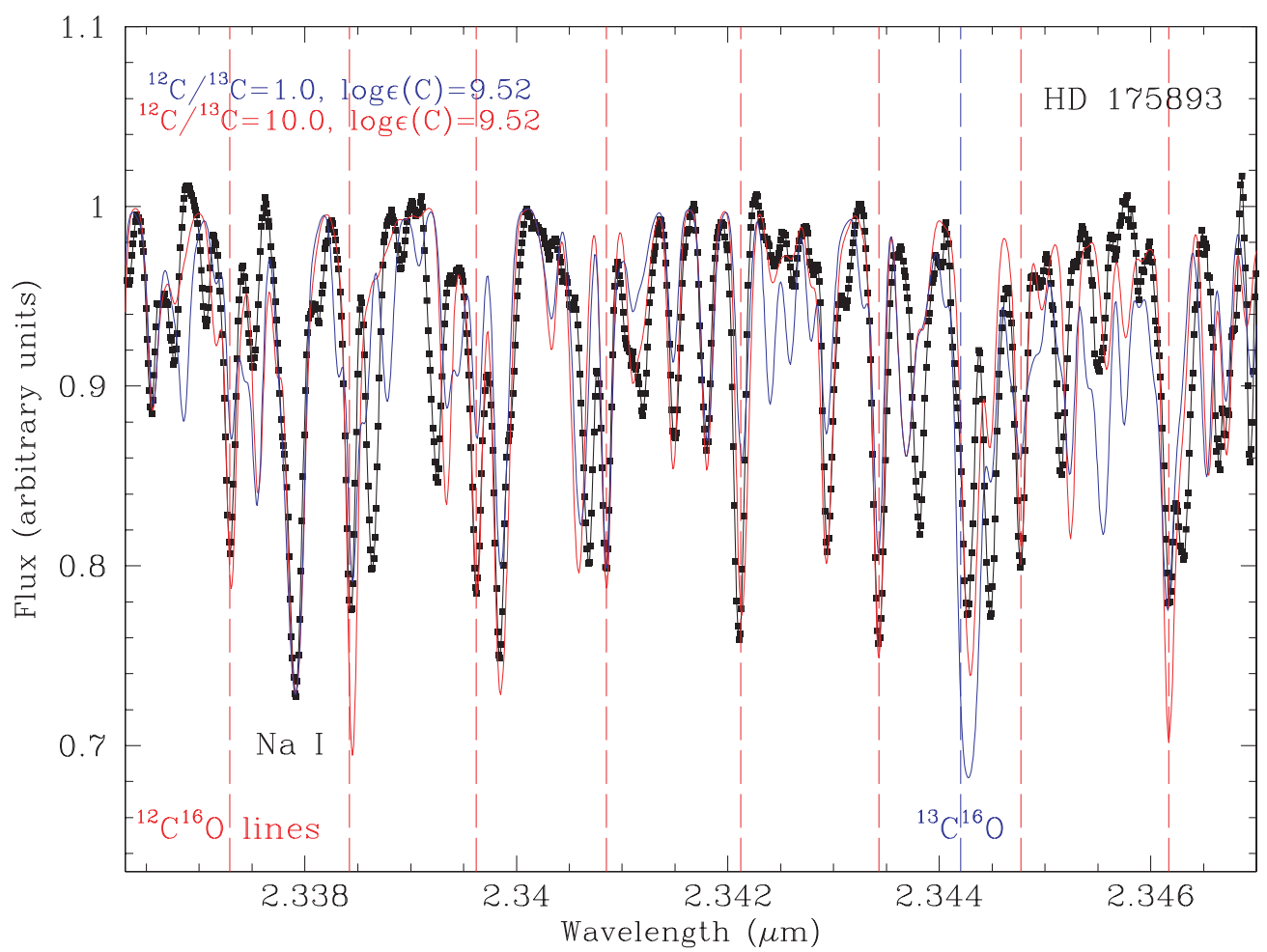

Figure 10. Best synthetic (red) and observed (black) spectrum in the region around the $2.344 \mu \mathrm{m}{ }^{13} \mathrm{C}^{16} \mathrm{O}$ bandhead for the ${ }^{18} \mathrm{O}$-rich $\mathrm{HdC}$ star $\mathrm{HD} 175893$. The red spectrum assumes the input $\mathrm{C}$ abundance of 9.52 , the $\mathrm{N}$ abundance of 9.2 , a total $\mathrm{O}\left({ }^{16} \mathrm{O}+{ }^{18} \mathrm{O}\right)$ of $\log \varepsilon(\mathrm{O})=8.7$, and the isotopic ratios ${ }^{16} \mathrm{O} /{ }^{18} \mathrm{O}$ and ${ }^{12} \mathrm{C} /{ }^{13} \mathrm{C}$ of 0.3 and 10.0, respectively. This region contains ${ }^{12} \mathrm{C}^{16} \mathrm{O}$ but not ${ }^{12} \mathrm{C}^{18} \mathrm{O}$ lines. The $2-0{ }^{13} \mathrm{C}^{16} \mathrm{O}$ bandhead is indicated with a blue dashed vertical line. The synthetic spectrum also includes $\mathrm{CN}$ lines. A synthetic spectrum (blue) for a lower ${ }^{12} \mathrm{C} /{ }^{13} \mathrm{C}$ ratio of 1.0 (or a higher ${ }^{13} \mathrm{C}$ abundance) but the same total $\mathrm{C}\left({ }^{12} \mathrm{C}+{ }^{13} \mathrm{C}\right.$ ) of $\log \varepsilon(C)=9.52$ is shown for comparison. Note the strong dependence of the $2-0{ }^{13} \mathrm{C}^{16} \mathrm{O}$ bandhead to changes of the ${ }^{12} \mathrm{C} /{ }^{13} \mathrm{C}$ ratio.

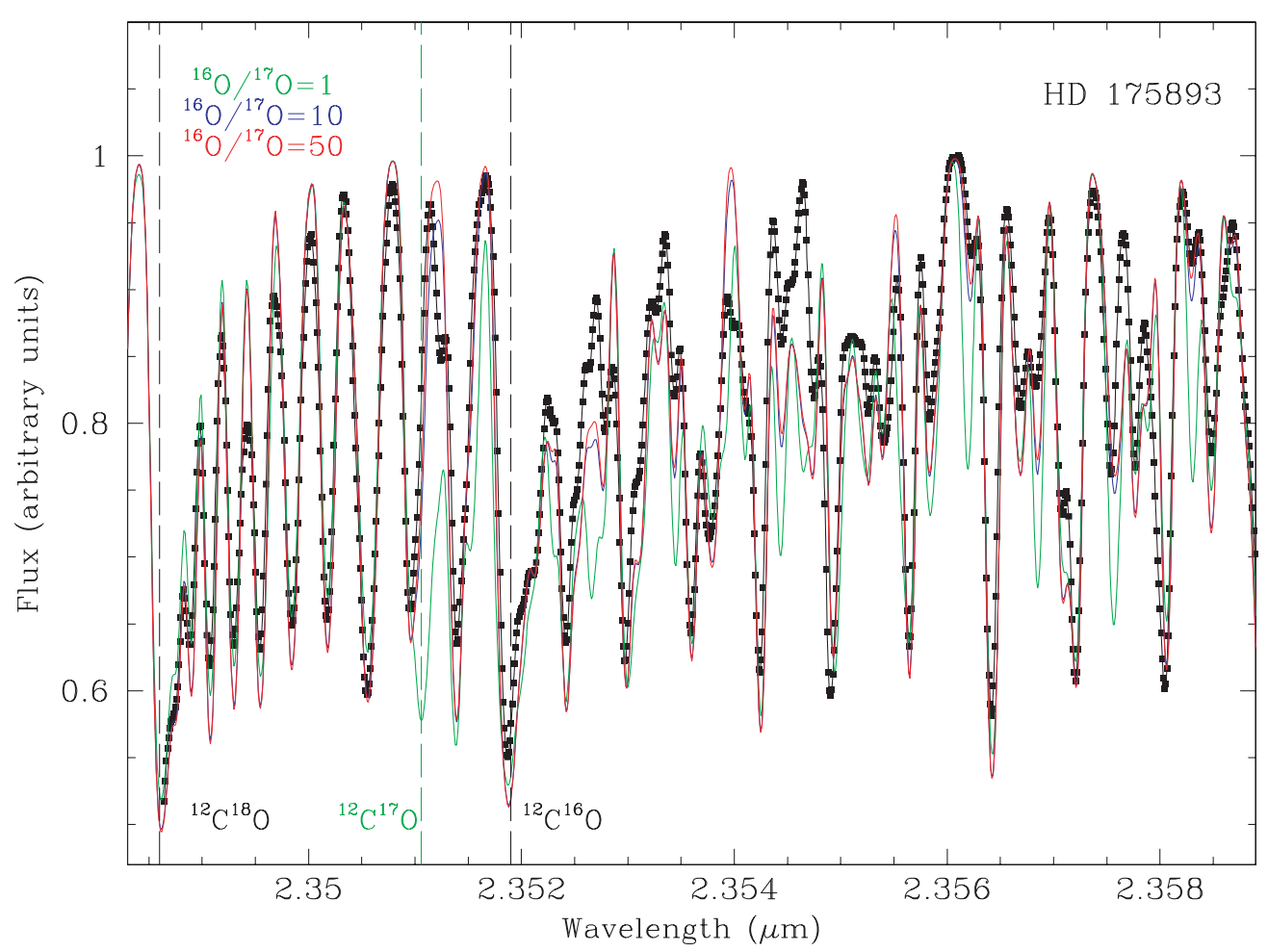

Figure 11. Best synthetic (red) and observed (black) spectrum in the region around the $2-02.349 \mu \mathrm{m}{ }^{12} \mathrm{C}^{18} \mathrm{O}$ bandhead for the ${ }^{18} \mathrm{O}-$-rich $\mathrm{HdC}$ star $\mathrm{HD}$ 175893. The spectrum also covers the expected position of the 3-1 $2.3511 \mu \mathrm{m}{ }^{12} \mathrm{C}^{17} \mathrm{O}$ bandhead. The red spectrum assumes the input $\mathrm{C}$ abundance of 9.52 , the $\mathrm{N}$ abundance of 9.2 , a total $\mathrm{O}\left({ }^{16} \mathrm{O}+{ }^{17} \mathrm{O}+{ }^{18} \mathrm{O}\right)$ of $\log \varepsilon(\mathrm{O})=8.7$ and the isotopic ratios ${ }^{16} \mathrm{O} /{ }^{18} \mathrm{O}=0.3$ and ${ }^{16} \mathrm{O} /{ }^{17} \mathrm{O}=50$. This region contains ${ }^{12} \mathrm{C}^{16} \mathrm{O}$ and ${ }^{12} \mathrm{C}^{18} \mathrm{O}$ lines which are fit with the isotopic ratio ${ }^{16} \mathrm{O} /{ }^{18} \mathrm{O}=0.3$. Synthetic spectra for lower ${ }^{16} \mathrm{O} /{ }^{17} \mathrm{O}$ ratios (or higher ${ }^{17} \mathrm{O}$ abundances) of 1 (green) and 10 (blue) but the same total $\mathrm{O}\left({ }^{16} \mathrm{O}+\right.$ ${ }^{17} \mathrm{O}+{ }^{18} \mathrm{O}$ ) of $\log \varepsilon(\mathrm{O})=8.7$ are shown for comparison. Note the strong dependence of the $3-12.3511 \mu \mathrm{m}^{12} \mathrm{C}^{17} \mathrm{O}$ bandhead (not detected in our spectra) to changes of the ${ }^{16} \mathrm{O} /{ }^{17} \mathrm{O}$ ratio. 


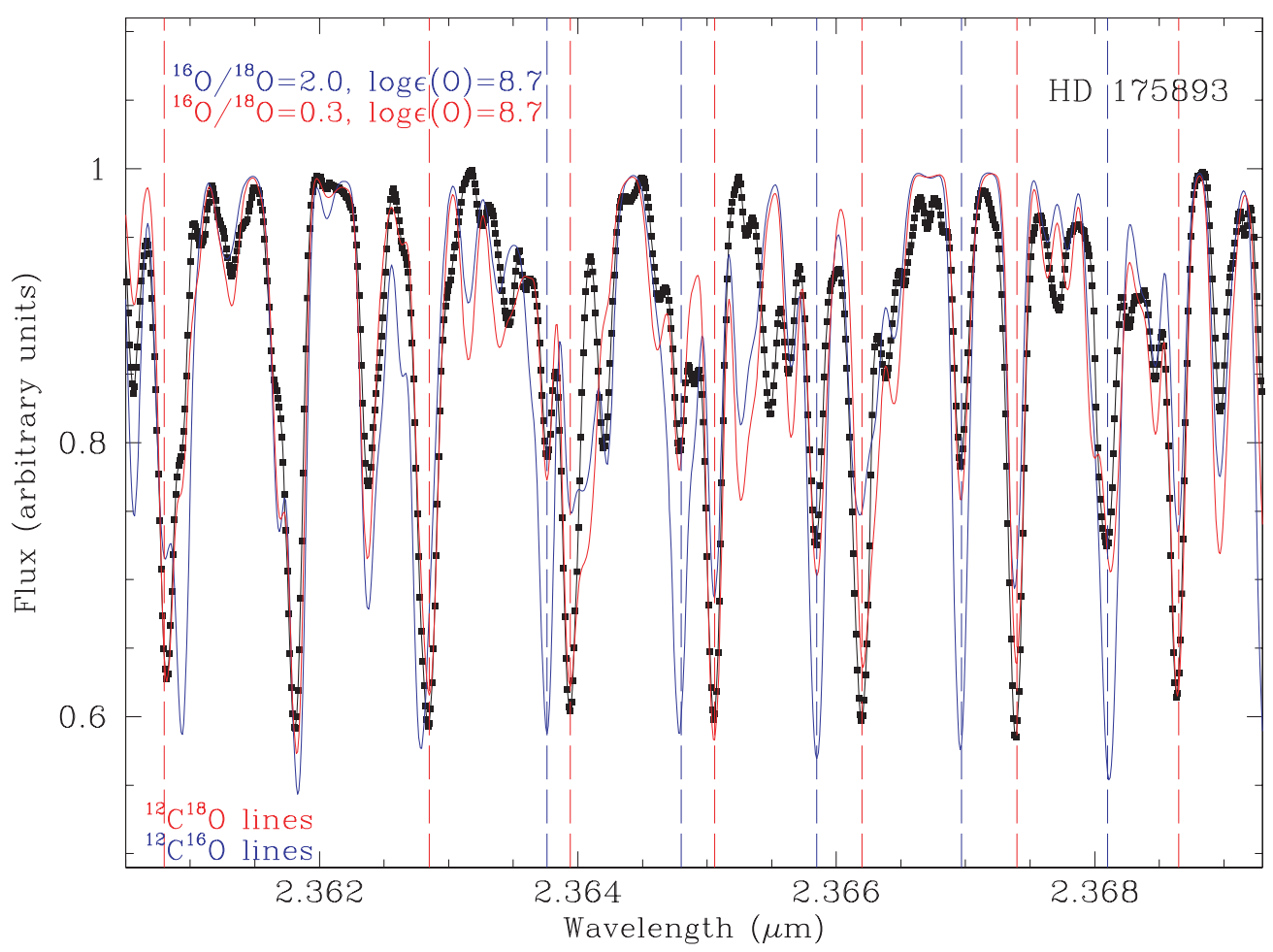

Figure 12. Best synthetic (red) and observed (black) spectrum for the region centered at $2.366 \mu \mathrm{m}$ for the ${ }^{18} \mathrm{O}-$-rich $\mathrm{HdC}$ star HD 175893 . The red spectrum assumes the input $\mathrm{C}$ abundance of 9.52 , the $\mathrm{N}$ abundance of 9.2 , a total $\mathrm{O}\left({ }^{16} \mathrm{O}+{ }^{18} \mathrm{O}\right)$ of $\log \varepsilon(\mathrm{O})=8.7$ and the isotopic ratio ${ }^{16} \mathrm{O} /{ }^{18} \mathrm{O}=0.3$. The most clear ${ }^{12} \mathrm{C}^{18} \mathrm{O}$ and ${ }^{12} \mathrm{C}^{16} \mathrm{O}$ lines are marked with red and blue vertical dashed lines, respectively. A synthetic spectrum (blue) for a higher ${ }^{16} \mathrm{O} /{ }^{18} \mathrm{O}$ ratio of 2 (or a lower ${ }^{18} \mathrm{O}$ abundance) but the same total $\mathrm{O}\left({ }^{16} \mathrm{O}+{ }^{18} \mathrm{O}\right)$ of $\log \varepsilon(\mathrm{O})=8.7$ is shown for comparison.

${ }^{12} \mathrm{C}^{17} \mathrm{O}$ and set the limit ${ }^{16} \mathrm{O} /{ }^{17} \mathrm{O}>50$, the first such estimate for an $\mathrm{HdC}$ star.

\subsection{2. $H D 137613$}

Analysis of the spectra followed the procedure just described for HD 175893. For the input $\mathrm{C}$ abundance $(=9.52)$, the $\mathrm{N}$ abundance from the $\mathrm{CN}$ lines is found to be $\log \epsilon(\mathrm{N})=9.4$ and the total $\mathrm{O}$ abundance to be $\log \epsilon(\mathrm{O})=8.7$. Selected comparisons of synthetic and observed spectra are provided in Figures 13, 14, and 15. With a ratio ${ }^{16} \mathrm{O} /{ }^{18} \mathrm{O}=0.5$, HD 137613 is slightly less ${ }^{18} \mathrm{O}$-rich than HD 175893 , a result consistent with Clayton et al.'s (2007) finding too. Our limit on the ${ }^{17} \mathrm{O}$ abundance is the same as for HD 175893. Our limit on ${ }^{12} \mathrm{C} /{ }^{13} \mathrm{C}(>10)$ is not competitive with determinations from CN Red System lines near $8000 \AA$ A: Kipper (2002) reported that "the ${ }^{12} \mathrm{C} /{ }^{13} \mathrm{C}$ ratio turns out be at least 90 " and Fujita \& Tsuji (1977) boldly set the ratio at 500 or greater. An absence of lines from ${ }^{13} \mathrm{C}$-containing molecules in optical spectra was noted by Warner (1967) for all five HdC stars.

\subsection{3. $H D 182040$}

Examination of Figure 1 shows that HD 182040 has CN lines of similar strength to those displayed by the twins HD 137613 and HD 175893. As mentioned in Section 3.1, our CN linelist is not complete. This incompleteness (wavelength errors, missing lines) is evident for HD 182040 where the $\mathrm{CN}$ molecule dominates the spectrum but an excellent fit to the observed spectrum cannot be obtained. Synthesis of the $2.251 \mu \mathrm{m}$ region (Figure 16) provides a satisfactory fit to the observed spectrum when the $\mathrm{N}$ abundance is $\log \epsilon\left({ }^{14} \mathrm{~N}\right)=9.2$ with the input $\mathrm{C}$ abundance $(=9.52)$ and the total $\mathrm{O}$ abundance set by the $\mathrm{CO}$ lines at $\log \epsilon(\mathrm{O})=8.0$. Again, we set the limit ${ }^{14} \mathrm{~N} /{ }^{15} \mathrm{~N}>10$.
Inspection of Figure 3, 4, and 7 shows that weak ${ }^{12} \mathrm{C}^{16} \mathrm{O}$ lines are present. Many other ${ }^{12} \mathrm{C}^{16} \mathrm{O}$ are obviously too strong; $\mathrm{CN}$ is the probable contaminant. In Figure 7, inspection similarly shows the presence of unblended ${ }^{12} \mathrm{C}^{18} \mathrm{O}$ lines with several others strengthened by blends. Unfortunately, the $2-0{ }^{12} \mathrm{C}^{18} \mathrm{O}$ bandhead was not completely captured on our spectra. We base our ${ }^{16} \mathrm{O} /{ }^{18} \mathrm{O}$ determination on the cleanest ${ }^{12} \mathrm{C}^{16} \mathrm{O}$ and ${ }^{12} \mathrm{C}^{18} \mathrm{O}$ lines (Figure 17). Anticipation of an distinctive $2-0{ }^{12} \mathrm{C}^{18} \mathrm{O}$ is strongly suggested by Figure 5. The ratio ${ }^{16} \mathrm{O} /{ }^{18} \mathrm{O}=0.5$ is obtained, a value consistent with the Clayton et al. (2007) estimate of 0.3 .

Our limit on the ${ }^{12} \mathrm{C} /{ }^{13} \mathrm{C}$ ratio $(>10)$ is consistent with the more stringent limit ${ }^{12} \mathrm{C} /{ }^{13} \mathrm{C}>100$ provided by Fujita \& Tsuji (1977). Our lower limit to the ${ }^{16} \mathrm{O} /{ }^{17} \mathrm{O}$ ratio is 8 .

\subsubsection{HD 173409}

Unfortunately, this star was not observed in the $2.251 \mu \mathrm{m}$ region and we could not obtain an estimate of the $\mathrm{N}$ abundance from a region free of $\mathrm{CO}$ lines. However, examination of our spectra (Figures 3 and 4) shows that weak ${ }^{12} \mathrm{C}^{14} \mathrm{~N}$ are clearly present in this star with $T_{\text {eff }}=6100 \mathrm{~K}$. Examination of the same figures shows that ${ }^{12} \mathrm{C}^{16} \mathrm{O}$ lines are weak, if not absent. A similar conclusion applies from Figure 7 to ${ }^{12} \mathrm{C}^{18} \mathrm{O}$ lines. Our spectra of HD 173409 did not capture the $2-0{ }^{12} \mathrm{C}^{18} \mathrm{O}$ bandhead at $2.349 \mu \mathrm{m}$ and at longer wavelengths the mix of ${ }^{12} \mathrm{C}^{16} \mathrm{O},{ }^{12} \mathrm{C}^{18} \mathrm{O}$ and ${ }^{12} \mathrm{C}^{14} \mathrm{~N}$ lines hampers identification of the $\mathrm{CO}$ isomers.

We have used the unblended ${ }^{12} \mathrm{C}^{14} \mathrm{~N}$ and possible ${ }^{12} \mathrm{C}^{16} \mathrm{O}$ features in the region $2.326-2.346 \mu \mathrm{m}$ to estimate the $\mathrm{N} / \mathrm{O}$ ratio (for the assumed $\mathrm{C}$ abundance). For this, we fixed the total $\mathrm{O}$ abundance to $\log \varepsilon(\mathrm{O})=8.7$ (as obtained for the $\mathrm{HdC}$ stars HD 137613 and HD 175893) and we calculated the ${ }^{14} \mathrm{~N}$ abundance needed to fit the cleanest ${ }^{12} \mathrm{C}^{14} \mathrm{~N}$ lines. We selected 


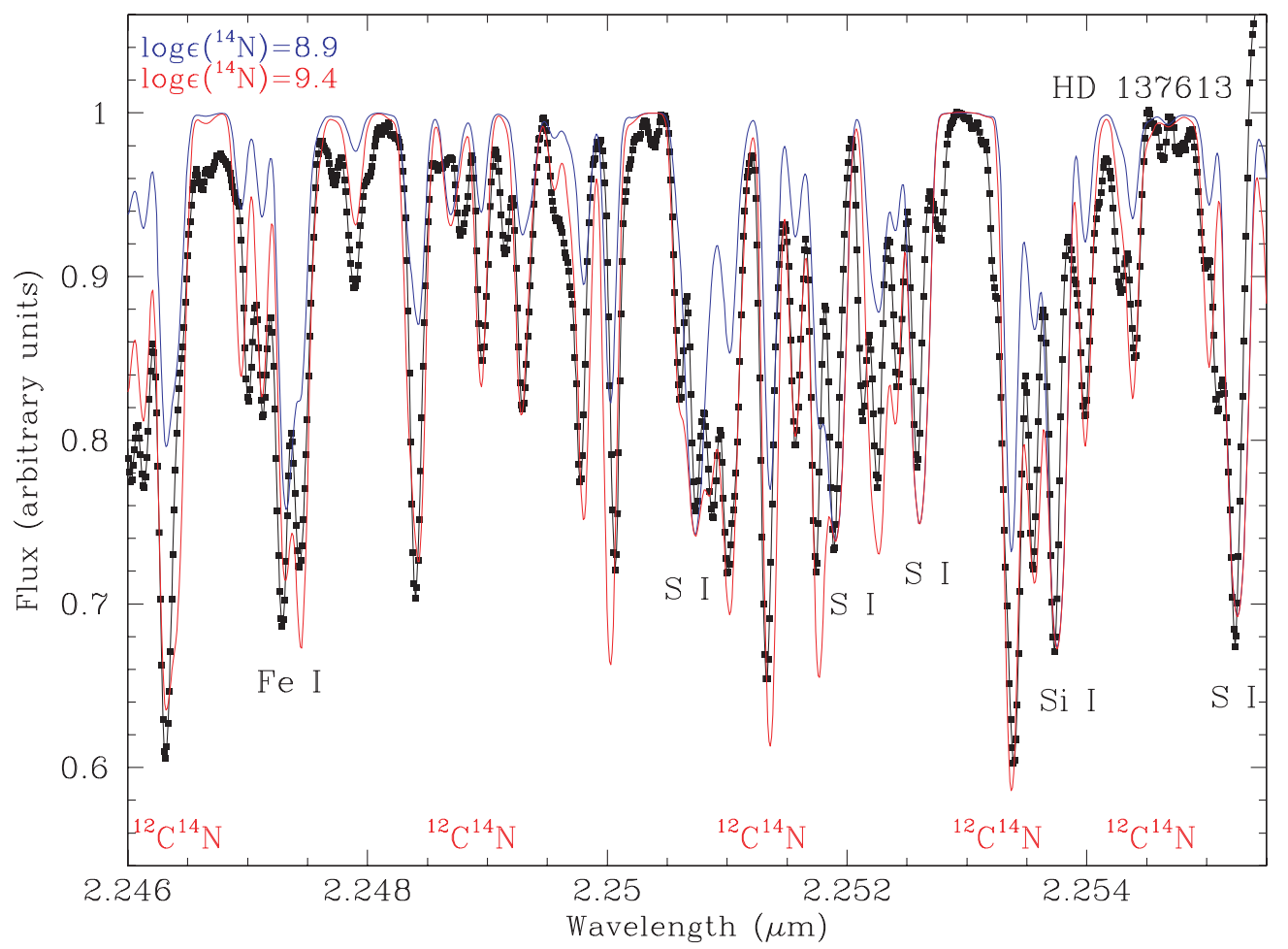

Figure 13. Best synthetic (red) and observed (black) spectrum for the $\mathrm{CN}$ region centered at $\sim 2.251 \mu \mathrm{m}$ for the ${ }^{18} \mathrm{O}-$-rich $\mathrm{HdC}$ star $\mathrm{HD} 137613$. The synthetic spectrum has been created assuming the input $\mathrm{C}$ abundance of 9.52 , the $\mathrm{N}$ is in the form of ${ }^{14} \mathrm{~N}$ with an abundance $\log \varepsilon(\mathrm{N})=9.4$ dex, and a total $\mathrm{O}$ abundance of $\log \varepsilon(\mathrm{O})=$ 8.7. A synthetic spectrum (blue) for a lower ${ }^{14} \mathrm{~N}$ abundance of $\log \varepsilon(\mathrm{N})=8.9$ dex is also shown for comparison.

(A color version of this figure is available in the online journal.)

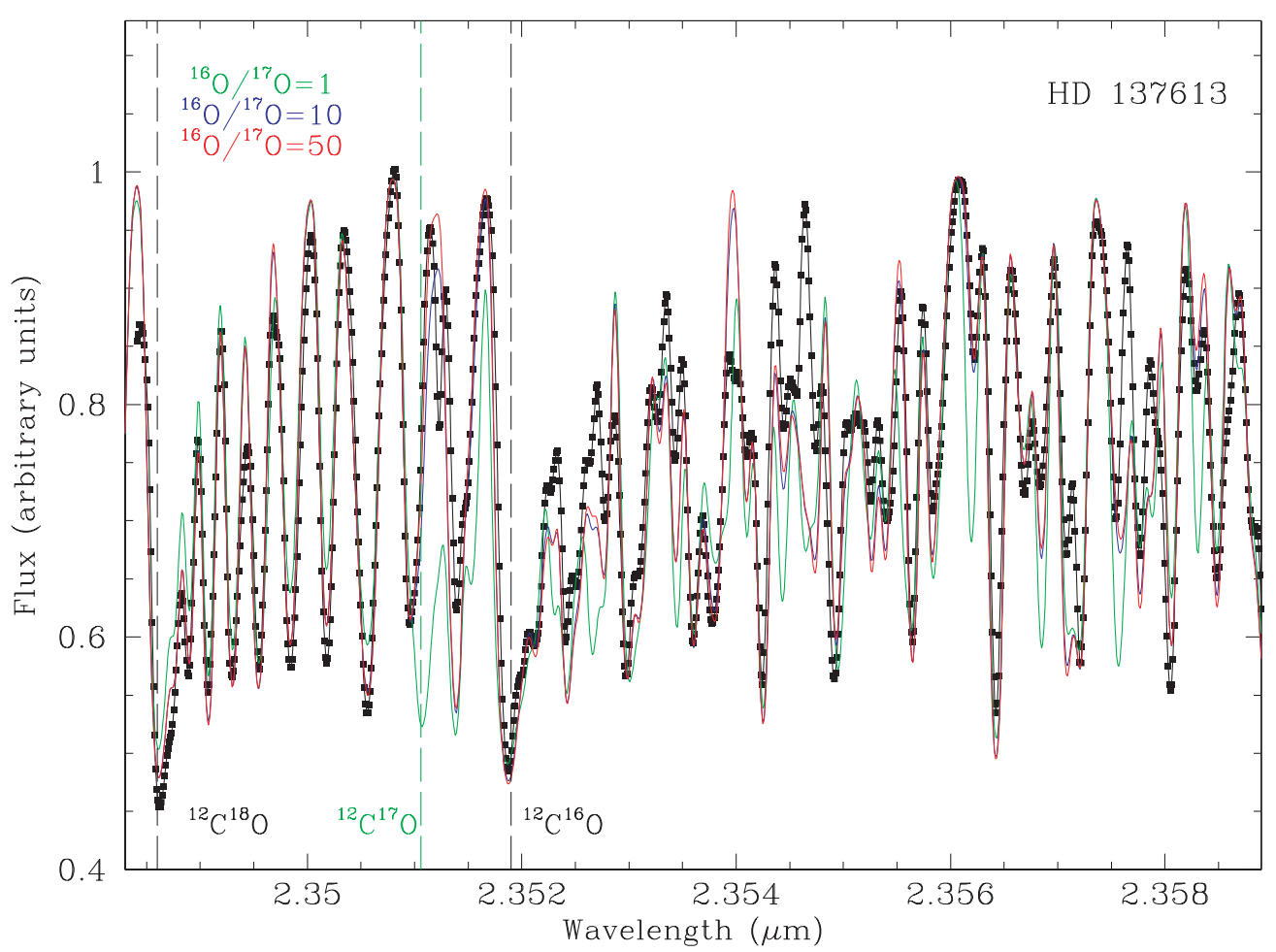

Figure 14. Best synthetic (red) and observed (black) spectrum in the region around the $2-02.349 \mu \mathrm{m}{ }^{12} \mathrm{C}^{18} \mathrm{O}$ bandhead for the ${ }^{18} \mathrm{O}-$-rich $\mathrm{HdC}$ star $\mathrm{HD} 137613$. The red spectrum assumes the input $\mathrm{C}$ abundance of 9.52 , the $\mathrm{N}$ abundance of 9.4 , a total $\mathrm{O}\left({ }^{16} \mathrm{O}+{ }^{17} \mathrm{O}+{ }^{18} \mathrm{O}\right)$ of $\log \varepsilon(\mathrm{O})=8.7$ and the isotopic ratios ${ }^{16} \mathrm{O} /{ }^{18} \mathrm{O}=0.5$ and ${ }^{16} \mathrm{O} /{ }^{17} \mathrm{O}=50$. Synthetic spectra for lower ${ }^{16} \mathrm{O} /{ }^{17} \mathrm{O}$ ratios (or higher ${ }^{17} \mathrm{O}$ abundances) of 1 (green) and 10 (blue) but the same total $\mathrm{O}\left({ }^{16} \mathrm{O}+{ }^{17} \mathrm{O}+{ }^{18} \mathrm{O}\right)$ of $\log \varepsilon(\mathrm{O})=$ 8.7 are shown for comparison. See Figure 11 for additional information.

(A color version of this figure is available in the online journal.) 


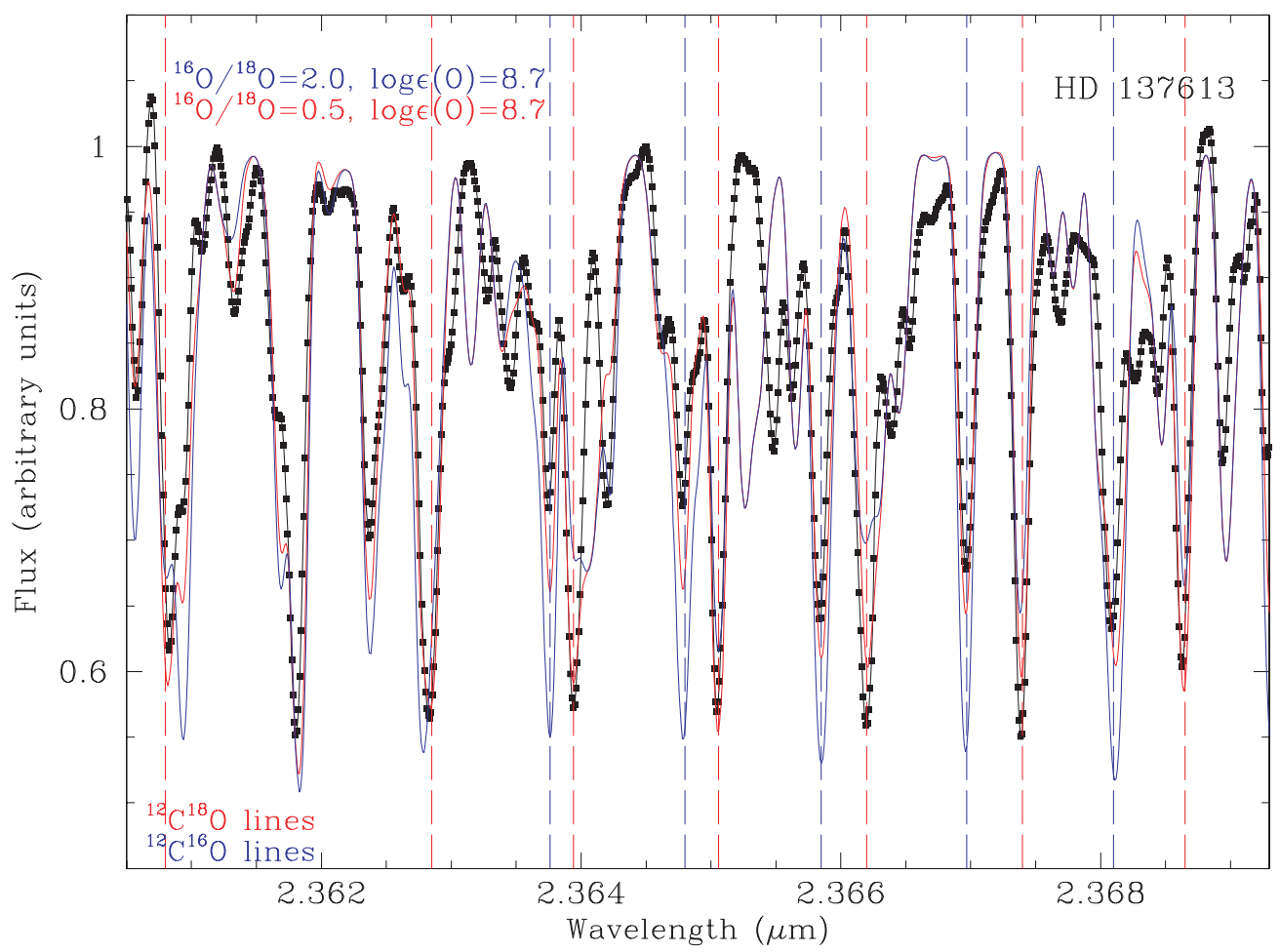

Figure 15. Best synthetic (red) and observed (black) spectrum for the region centered at $2.366 \mu \mathrm{m}$ for the ${ }^{18} \mathrm{O}$-rich $\mathrm{HdC}$ star HD 137613. The red spectrum assumes the input $\mathrm{C}$ abundance of 9.52 , the $\mathrm{N}$ abundance of 9.4 , a total $\mathrm{O}\left({ }^{16} \mathrm{O}+{ }^{18} \mathrm{O}\right)$ of $\log \varepsilon(\mathrm{O})=8.7$ and the isotopic ratio ${ }^{16} \mathrm{O} /{ }^{18} \mathrm{O}=0.5$. Some of the strongest ${ }^{12} \mathrm{C}^{18} \mathrm{O}$ and ${ }^{12} \mathrm{C}^{16} \mathrm{O}$ lines are marked with red and blue vertical dashed lines, respectively. A synthetic spectrum (blue) for a higher ${ }^{16} \mathrm{O} /{ }^{18} \mathrm{O}$ ratio of 2 (or a lower ${ }^{18} \mathrm{O}$ abundance) but the same total $\mathrm{O}\left({ }^{16} \mathrm{O}+{ }^{18} \mathrm{O}\right)$ of $\log \varepsilon(\mathrm{O})=8.7$ is shown for comparison.

(A color version of this figure is available in the online journal.)

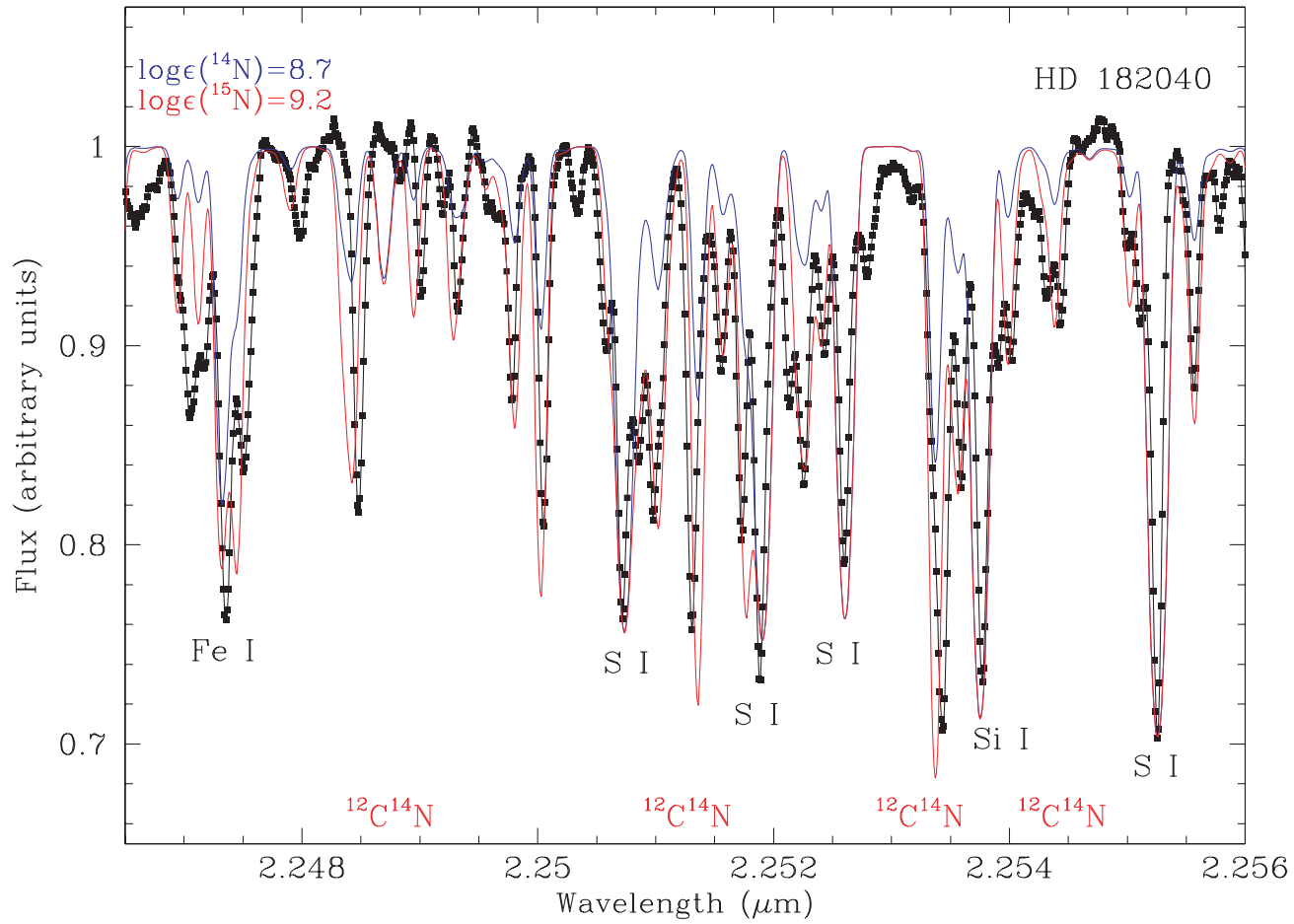

Figure 16. Best synthetic (red) and observed (black) spectrum for the $\mathrm{CN}$ region centered at $\sim 2.251 \mu \mathrm{m}$ for the ${ }^{18} \mathrm{O}-$-rich $\mathrm{HdC}$ star HD 182040 . The synthetic spectrum has been created assuming the input $\mathrm{C}$ abundance of 9.52 , the $\mathrm{N}$ is in the form of ${ }^{14} \mathrm{~N}$ with an abundance $\log \varepsilon(\mathrm{N})=9.2$ dex, and a total $\mathrm{O}$ abundance of $\log \varepsilon(\mathrm{O})=$ 8.0. A synthetic spectrum (blue) for a lower ${ }^{14} \mathrm{~N}$ abundance of $\log \varepsilon(\mathrm{N})=8.7$ dex is also shown for comparison.

(A color version of this figure is available in the online journal.)

a few ${ }^{12} \mathrm{C}^{14} \mathrm{~N}$ lines (e.g., those around $2.342 \mu \mathrm{m}$; see Figure 18) for which a perfect agreement with the $\mathrm{N}$ abundance derived from the $2.251 \mu \mathrm{m}$ region was obtained in the other $\mathrm{HdC}$ stars. By this procedure, the $\mathrm{N}$ abundance from the $\mathrm{CN}$ lines is found 


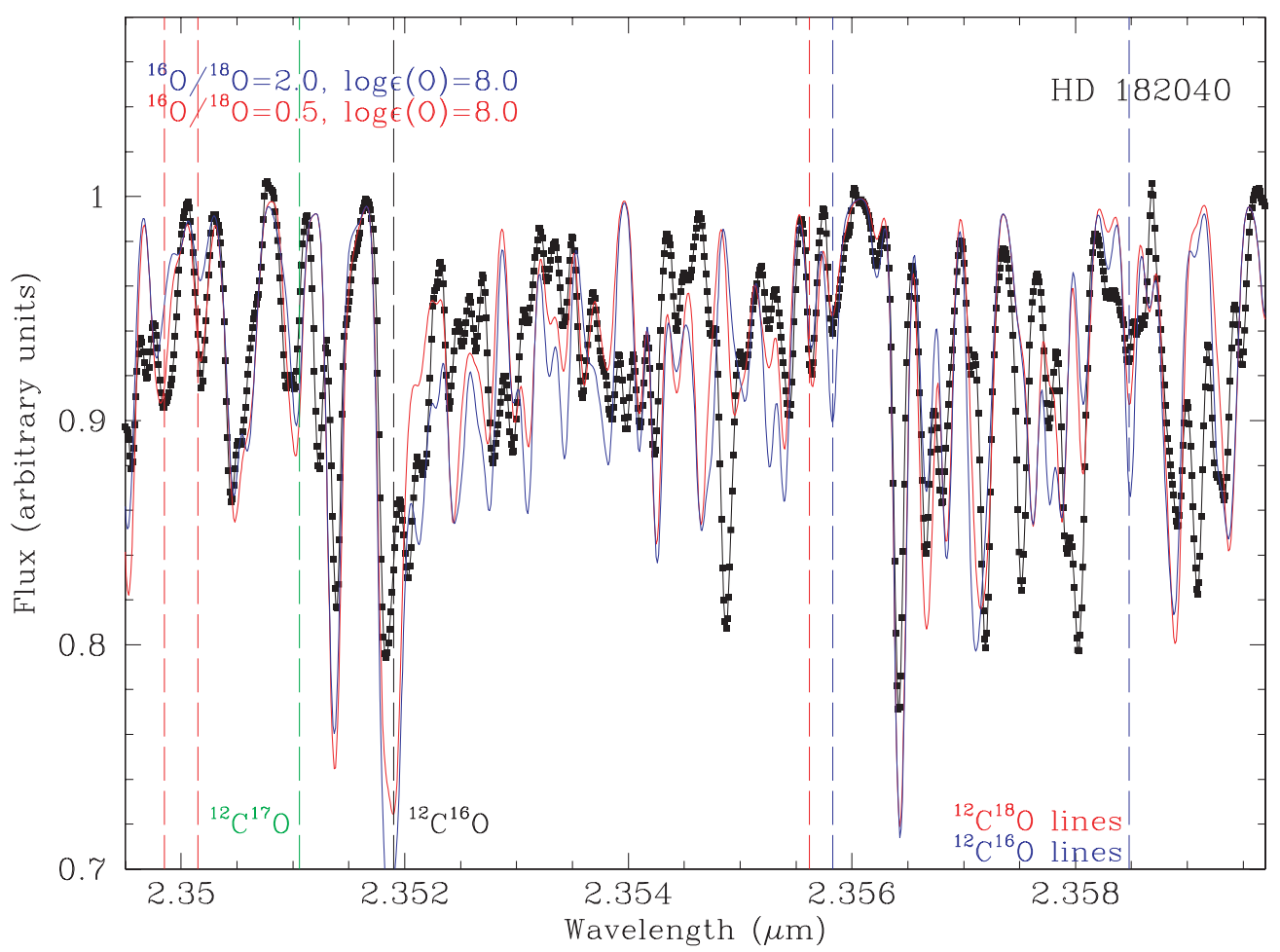

Figure 17. Best synthetic (red) and observed (black) spectrum in the region around the $2-02.349 \mu \mathrm{m}{ }^{12} \mathrm{C}^{18} \mathrm{O}$ bandhead for the ${ }^{18} \mathrm{O}$-rich $\mathrm{HdC}$ star $\mathrm{HD} 182040$. The red spectrum assumes the input $\mathrm{C}$ abundance of 9.52 , the $\mathrm{N}$ abundance of 9.2 , a total $\mathrm{O}\left({ }^{16} \mathrm{O}+{ }^{18} \mathrm{O}\right)$ of $\log \varepsilon(\mathrm{O})=8.0$ and the isotopic ratio ${ }^{16} \mathrm{O} /{ }^{18} \mathrm{O}=0.5$. A synthetic spectrum for a different ${ }^{16} \mathrm{O} /{ }^{18} \mathrm{O}$ ratio of 2 (blue) but the same total $\mathrm{O}\left({ }^{16} \mathrm{O}+{ }^{18} \mathrm{O}\right)$ of $\log \varepsilon(\mathrm{O})=8.0$ is shown for comparison. Note that there are several features (e.g., those at $\sim 2.3540,2.3550,2.3575,2.3580$, and $2.3590 \mu \mathrm{m}$ ) which are not reproduced by our synthetic spectra.

(A color version of this figure is available in the online journal.)

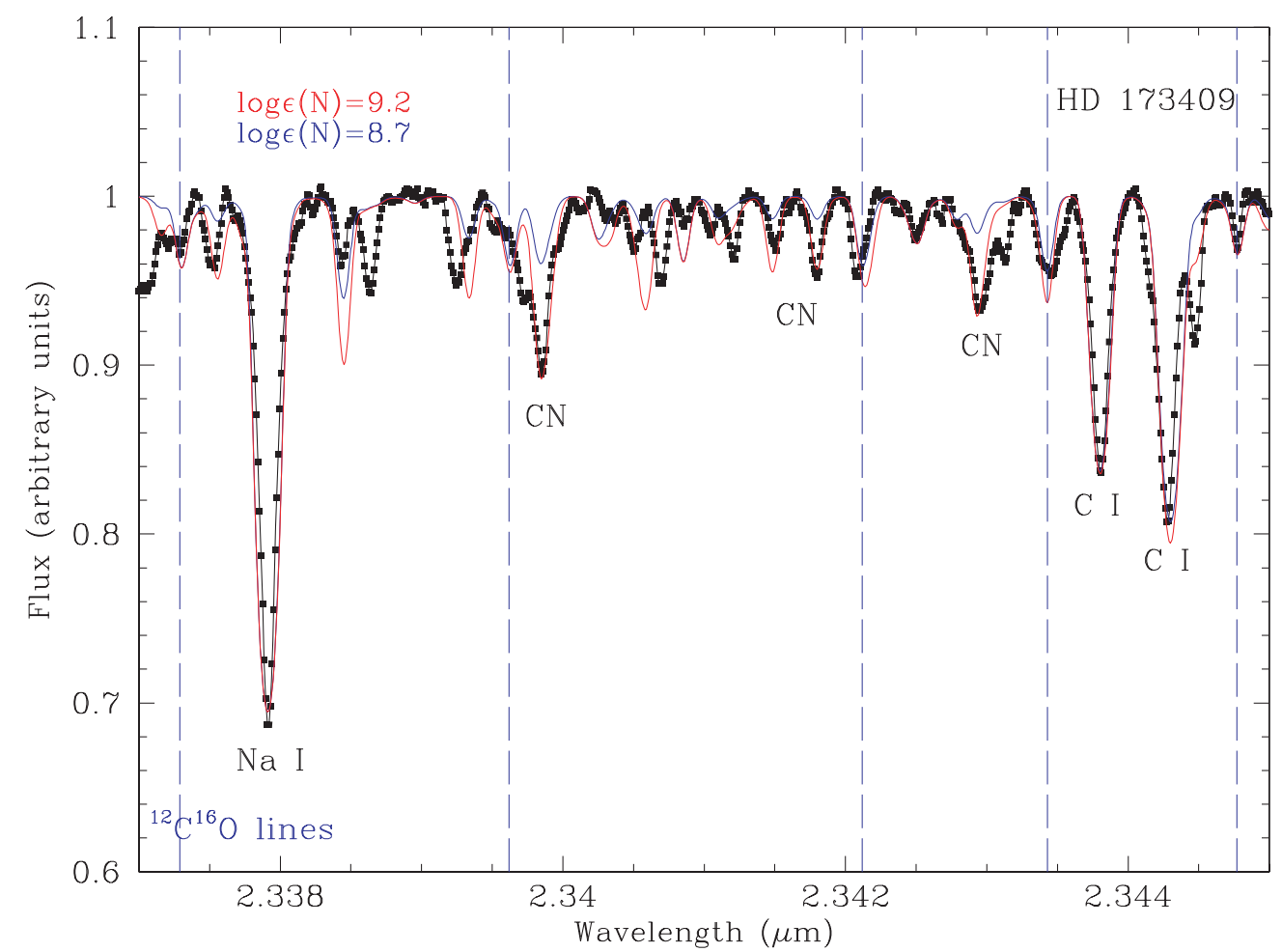

Figure 18. Best synthetic (red) and observed (black) spectrum for the $\mathrm{CO}$ and $\mathrm{CN}$ region centered at $\sim 2.341 \mu \mathrm{m}$ for the HdC star HD 173409 . The synthetic spectrum has been created assuming the input $\mathrm{C}$ abundance of 9.52 , the $\mathrm{N}$ is in the form of ${ }^{14} \mathrm{~N}$ with an abundance $\log \varepsilon(\mathrm{N})=9.2$ dex, and a total $\mathrm{O}$ abundance fixed at $\log \varepsilon(\mathrm{O})=8.7$. A synthetic spectrum for a lower ${ }^{14} \mathrm{~N}$ content of $\log \varepsilon(\mathrm{N})=8.7$ (in blue) is also shown for comparison.

(A color version of this figure is available in the online journal.) 


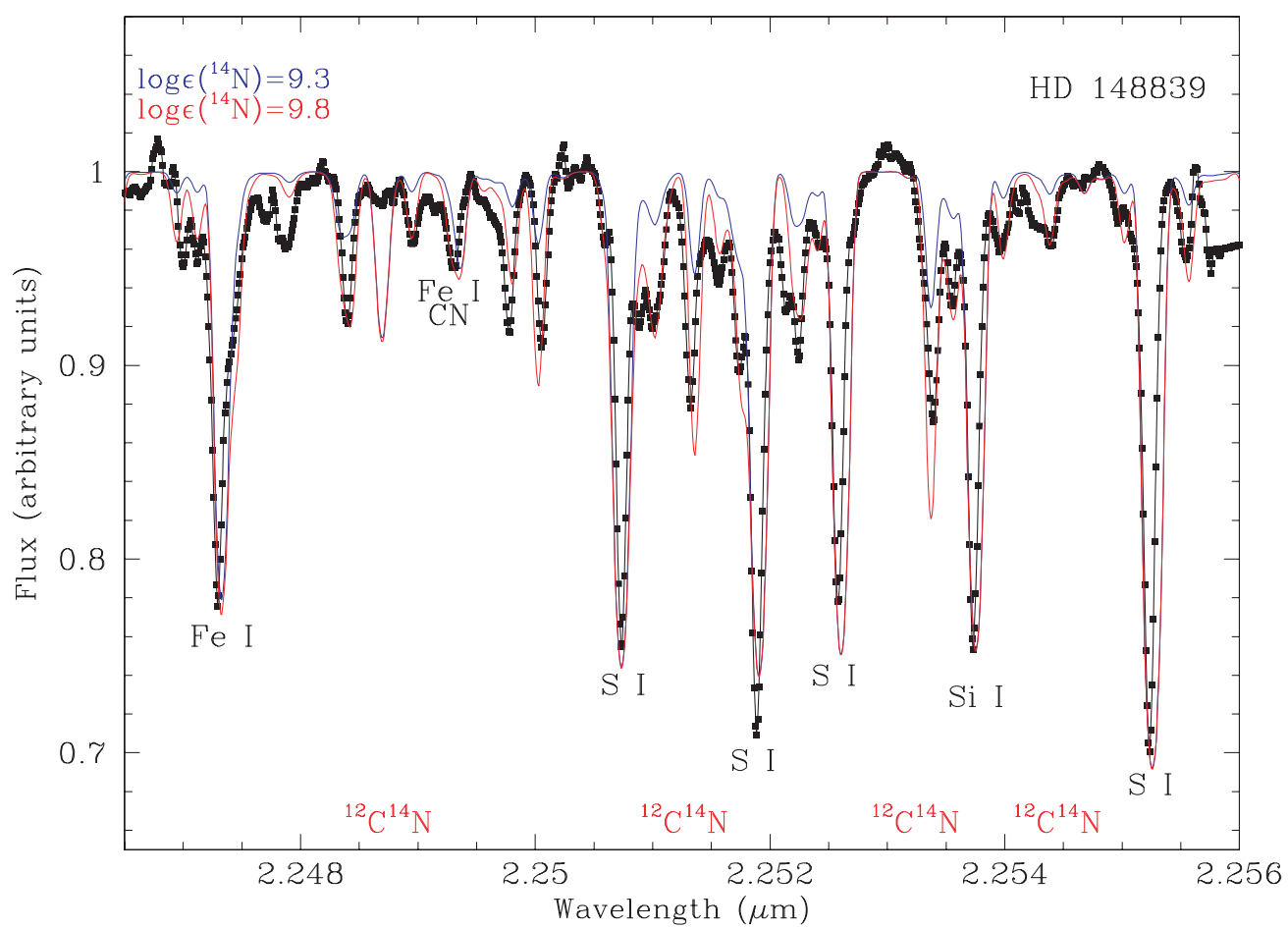

Figure 19. Best synthetic (red) and observed (black) spectrum for the $\mathrm{CN}$ region centered at $\sim 2.251 \mu \mathrm{m}$ for the HdC star HD 148839. The synthetic spectrum has been created assuming the input $\mathrm{C}$ abundance of 9.52 , the $\mathrm{N}$ is in the form of ${ }^{14} \mathrm{~N}$ with an abundance $\log \varepsilon(\mathrm{N})=9.8$ dex, and a total $\mathrm{O}$ abundance of $\log \varepsilon(\mathrm{O})=9.2$. $\mathrm{A}$ synthetic spectrum (blue) for a lower ${ }^{14} \mathrm{~N}$ abundance of $\log \varepsilon(\mathrm{N})=9.3 \mathrm{dex}$ is also shown for comparison.

(A color version of this figure is available in the online journal.)

to be $\log \epsilon(\mathrm{N})=9.2$, giving a N/O ratio of $\log \epsilon(\mathrm{N} / \mathrm{O})=+0.5$. Through a family of syntheses, we conclude that determination of the ${ }^{16} \mathrm{O} /{ }^{18} \mathrm{O}$ ratio is very difficult, if not impossible, to determine from the present observations.

\subsubsection{HD 148839}

In spite of the higher effective temperature $(6500 \mathrm{~K})$ for this star, the CN lines are of similar strength to those of HD 173409 $(6100 \mathrm{~K})$. The strongest ${ }^{12} \mathrm{C}^{14} \mathrm{~N}$ features are clearly seen in the $2.251 \mu \mathrm{m}$ spectrum (Figures 1 and 2). Our spectrum synthesis in this region (Figure 19) gives a high $\mathrm{N}$ abundance of 9.8 with the input $\mathrm{C}$ abundance $(=9.52)$ and a total $\mathrm{O}$ abundance of 9.2, the best estimate from tentative identifications of $\mathrm{CO}$ lines.

Inspection of Figures 3 and 4 suggests that ${ }^{12} \mathrm{C}^{16} \mathrm{O}$ lines are weak but consistently present. Evidence for ${ }^{12} \mathrm{C}^{18} \mathrm{O}$ lines is less certain. One may contrast the two stars HD 148839 and HD 182040: ${ }^{12} \mathrm{C}^{16} \mathrm{O}$ lines appear present in HD 148839 and weaker in HD 182040 with the opposite being the case for the ${ }^{12} \mathrm{C}^{18} \mathrm{O}$ lines. The lower limit for the ratio ${ }^{16} \mathrm{O} /{ }^{18} \mathrm{O}$ is probably 2 . This is intriguing because HD 148839 is Li-rich (Rao \& Lambert 1996) and synthesis of ${ }^{18} \mathrm{O}$ resulting from the DD merger would seem difficult to reconcile with the synthesis of $\mathrm{Li}$ (see below).

$$
\text { 3.2.6. S Aps }
$$

The CN lines are of similar strength to those in HD 137613 and HD 175893. The synthetic spectrum fitted to $\mathrm{CN}$ lines in the $2.251 \mu \mathrm{m}$ region (Figure 21) gives a $\mathrm{N}$ abundance of 9.6 with the input $\mathrm{C}$ abundance $(=9.52)$ and the total $\mathrm{O}$ abundance $(=9.4)$ from the CO lines. Synthesis of the $2.354 \mu \mathrm{m}$ region gives a good overall fit to the observed spectrum (Figure 22). The lower limits on the ${ }^{14} \mathrm{~N} /{ }^{15} \mathrm{~N}(>10)$, and ${ }^{16} \mathrm{O} /{ }^{17} \mathrm{O}(>80)$ ratios are comparable to those set for HD 137613 and HD 175893. We could not set a lower limit on the ${ }^{12} \mathrm{C} /{ }^{13} \mathrm{C}$ ratio because this star was not observed around the $2-0{ }^{13} \mathrm{C}^{16} \mathrm{O}$ bandhead at $2.344 \mu \mathrm{m}$.

Inspection of Figures 6 and 22 shows that the ${ }^{16} \mathrm{O} /{ }^{18} \mathrm{O}$ ratio for $\mathrm{S}$ Aps is greater than unity in contrast to the values of less than unity prevailing in HD 137613 and HD 175893. The derived isotopic ratio is ${ }^{16} \mathrm{O} /{ }^{18} \mathrm{O}=16$, a value larger than the Clayton et al. (2007) estimate of 4. Our result is somewhat dependent on the adopted microturbulence given that the available ${ }^{12} \mathrm{C}^{18} \mathrm{O}$ lines are systematically weaker than the ${ }^{12} \mathrm{C}^{16} \mathrm{O}$ lines. S Aps is the only $\mathrm{RCB}$ star in our sample for which the isotopic $\mathrm{O}$ composition could be measured. This is unfortunate because $\mathrm{S}$ Aps possibly offers the hint that the RCBs may be less enriched in ${ }^{18} \mathrm{O}$ than the $\mathrm{HdCs}$.

\subsection{Elemental Abundances- $C, N$, and $O$}

For the analysis of the $\mathrm{CN}$ and $\mathrm{CO}$ lines, we assumed that the $\mathrm{C}$ abundance is equal to the input $\mathrm{C}$ abundance of the adopted model atmosphere, which for models with $\mathrm{C} / \mathrm{He}=1 \%$ is a $\mathrm{C}$ abundance of 9.52. As long as the assumed $\mathrm{C}$ abundance is greater than the total $\mathrm{O}\left({ }^{16} \mathrm{O}+{ }^{18} \mathrm{O}\right)$, the $\mathrm{CO}$ number density is not very dependent on the $\mathrm{C}$ abundance. In the case of $\mathrm{CN}$, the number density is dependent on the difference between the $\mathrm{C}$ and $\mathrm{O}$ abundances thanks to the dominant role of $\mathrm{CO}$ formation, and on the $\mathrm{N}$ abundance with $\mathrm{N}_{2}$ playing the controlling role in setting the partial pressure of $\mathrm{N}$. The abundance of $\mathrm{C}$ (i.e., the $\mathrm{C} / \mathrm{He}$ ratio) plays a secondary role in setting the continuous opacity.

A check on the input $\mathrm{C}$ abundance is possible from the $\mathrm{C}$ I lines. Both $\mathrm{C}_{\mathrm{I}}$ lines are usable in the two hottest stars HD 173409 and HD 148839. Unfortunately, the $\mathrm{C}_{\mathrm{I}}$ line at $2.3443 \mu \mathrm{m}$ is strongly blended with molecular features in the cooler HdC stars although the other $\mathrm{C}$ I line at $2.3438 \mu \mathrm{m}$, if correctly identified, 


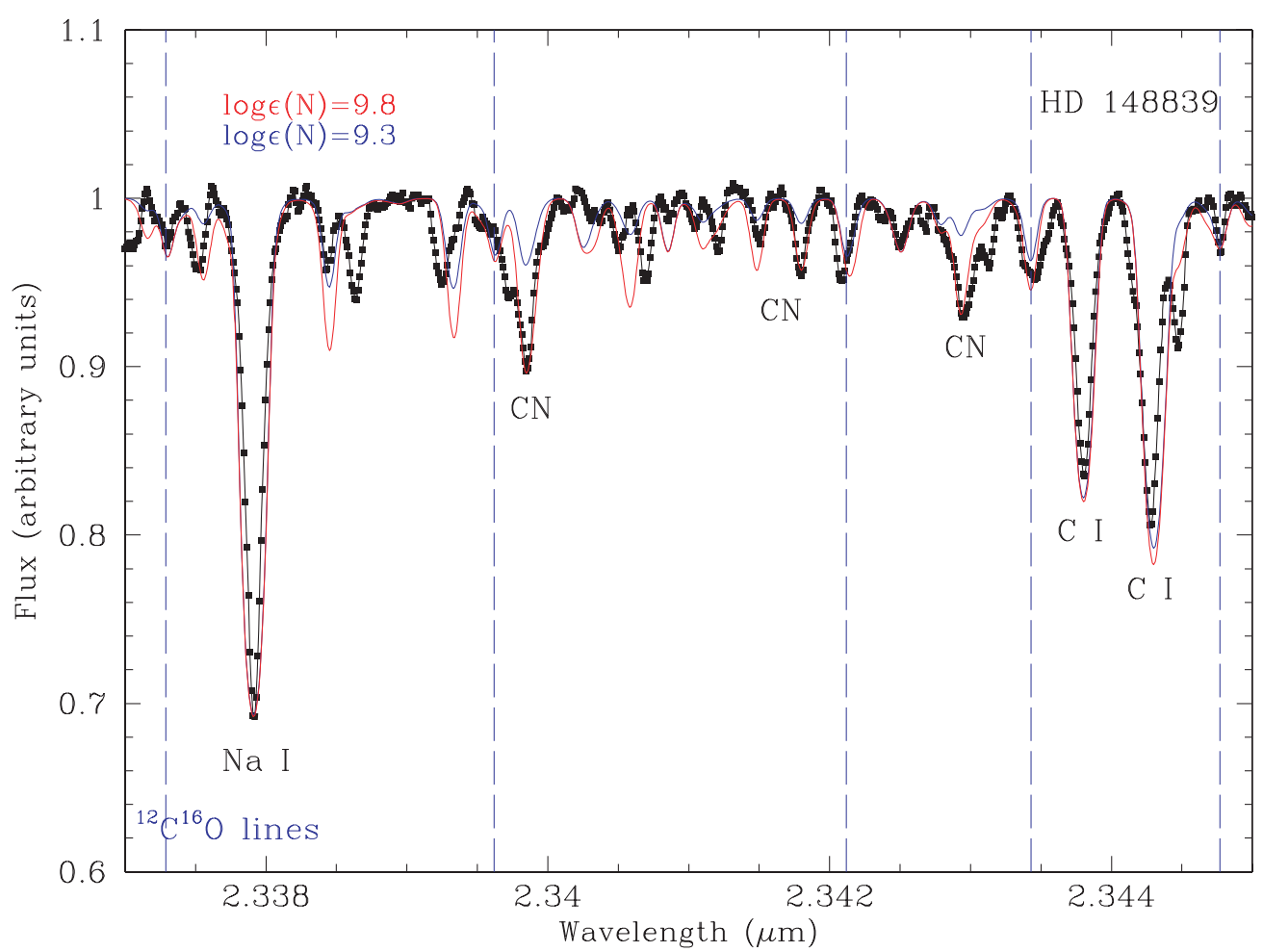

Figure 20. Best synthetic (red) and observed (black) spectrum for the $\mathrm{CO}$ and $\mathrm{CN}$ region centered at $\sim 2.341 \mu \mathrm{m}$ for the HdC star HD 148839. The synthetic spectrum has been created assuming the input $\mathrm{C}$ abundance of 9.52 , the $\mathrm{N}$ is in the form of ${ }^{14} \mathrm{~N}$ with an abundance $\log \varepsilon(\mathrm{N})=9.8$ dex, and a total $\mathrm{O}$ abundance of $\log \varepsilon(\mathrm{O})=$ 9.2. A synthetic spectrum for a lower ${ }^{14} \mathrm{~N}$ abundance of $\log \varepsilon(\mathrm{N})=9.3$ (in blue) is also shown for comparison.

(A color version of this figure is available in the online journal.)

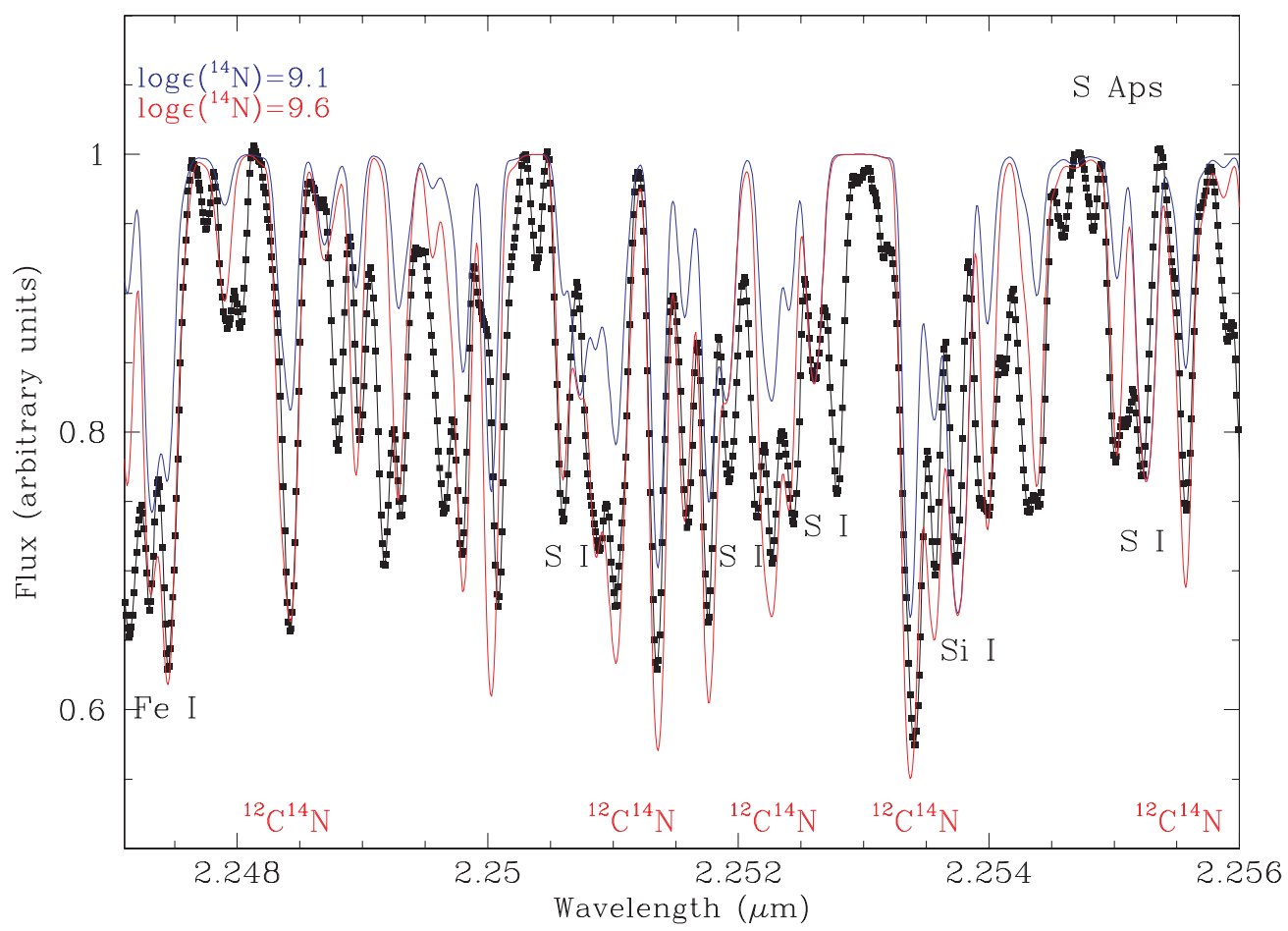

Figure 21. Best synthetic (red) and observed (black) spectrum for the $\mathrm{CN}$ region centered at $\sim 2.251 \mu \mathrm{m}$ for the ${ }^{18} \mathrm{O}-$ rich RCB star $\mathrm{S}$ Aps. The synthetic spectrum has been created assuming the input $\mathrm{C}$ abundance of 9.52 , the $\mathrm{N}$ is in the form of ${ }^{14} \mathrm{~N}$ with an abundance $\log \varepsilon(\mathrm{N})=9.6 \mathrm{dex}$, and a total $\mathrm{O}$ abundance of $\log \varepsilon(\mathrm{O})=9.4$. $\mathrm{A}$ synthetic spectrum (blue) for a lower ${ }^{14} \mathrm{~N}$ abundance of $\log \varepsilon(\mathrm{N})=9.1$ dex is also shown for comparison.

(A color version of this figure is available in the online journal.)

seems to be unblended. The wavelength interval providing the C I lines was not observed for S Aps and the other RCB stars.
Analysis of the five $\mathrm{HdC}$ stars using models computed for $\mathrm{C} / \mathrm{He}=1 \%$, i.e., an assumed abundance $\log \epsilon(\mathrm{C})=9.52$, provide 


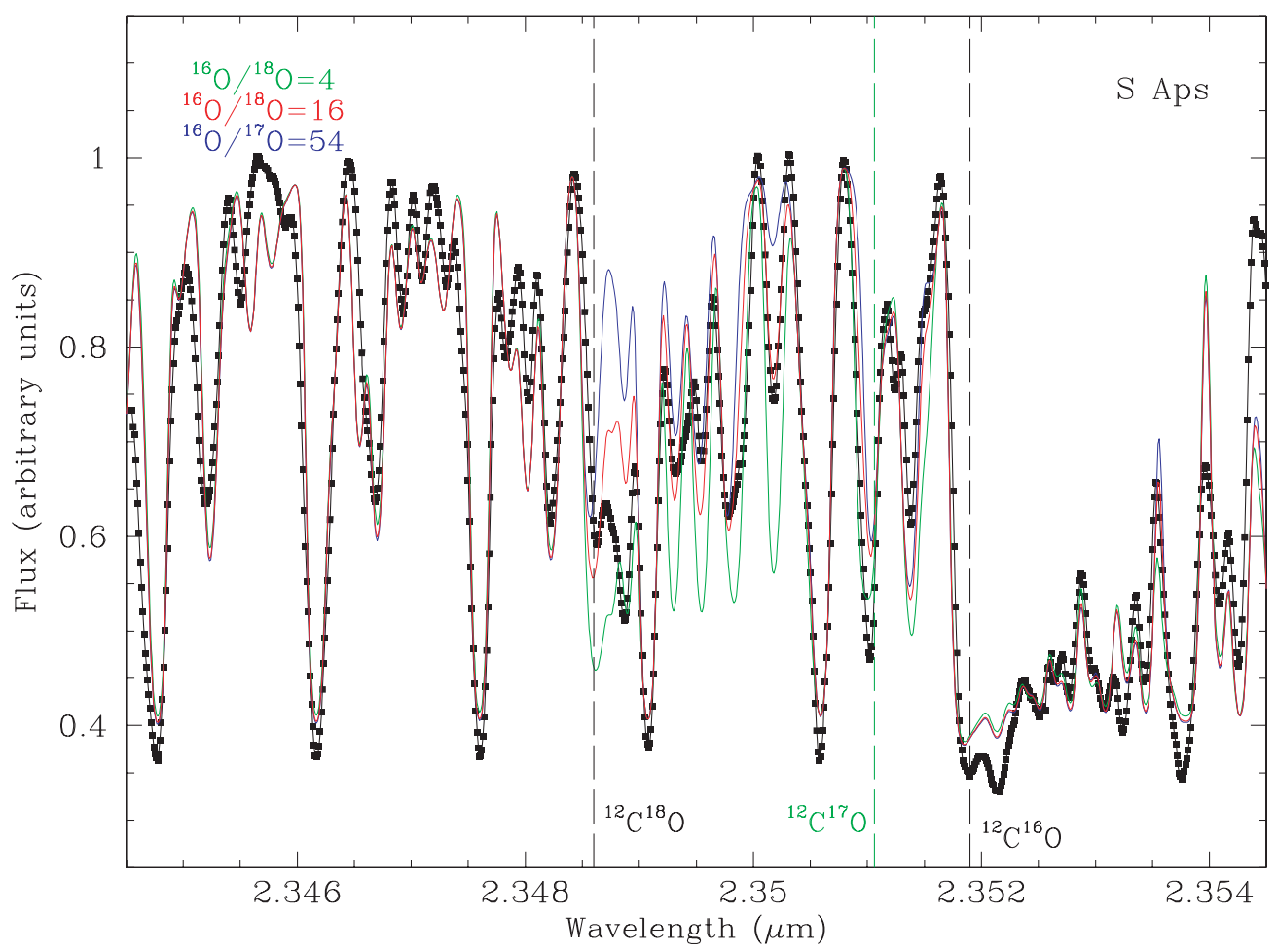

Figure 22. Best synthetic (red) and observed (black) spectrum in the region around the $2-02.349 \mu \mathrm{m}{ }^{12} \mathrm{C}^{18} \mathrm{O}$ bandhead for the ${ }^{18} \mathrm{O}$-rich $\mathrm{RCB}$ star $\mathrm{S}$ Aps. The red spectrum assumes the input $\mathrm{C}$ abundance of 9.52 , the $\mathrm{N}$ abundance of 9.6 , a total $\mathrm{O}\left({ }^{16} \mathrm{O}+{ }^{18} \mathrm{O}\right)$ of $\log \varepsilon(\mathrm{O})=9.4$ and the isotopic ratio ${ }^{16} \mathrm{O} /{ }^{18} \mathrm{O}=16$. Synthetic spectra for different ${ }^{16} \mathrm{O} /{ }^{18} \mathrm{O}$ ratios of 4 (green) and 54 (blue) but the same total $\mathrm{O}\left({ }^{16} \mathrm{O}+{ }^{18} \mathrm{O}\right)$ of $\log \varepsilon(\mathrm{O})=9.4$ are shown for comparison.

(A color version of this figure is available in the online journal.)

from the $\mathrm{C}$ I lines carbon abundances that average only +0.1 dex greater than the assumed abundance with a range from -0.3 to +0.4 dex. The extremes of the range are provided by HD 137613 at -0.3 dex and HD 182040 at +0.4 dex. This is a satisfactory level of consistency between the $\mathrm{C}$ abundance adopted in the models' construction and that provided from the $\mathrm{C}$ I line(s). Thus, the $\mathrm{C}$ I lines do not exhibit the carbon problem highlighted by Asplund et al. (2000) in their analysis of the optical spectra of RCBs. The $\mathrm{C}$ abundance obtained depends on the $\mathrm{C} / \mathrm{He}$ ratio adopted for the models. At $\mathrm{C} / \mathrm{He}=10 \%$, the $\mathrm{C}$ abundance from $\mathrm{C}_{\mathrm{I}}$ lines is about 0.2 dex larger. For $\mathrm{C} / \mathrm{He}<1 \%$ models, there is a point at which the input $\mathrm{C}$ abundance is less than the $\mathrm{O}$ abundance needed to account for the CO lines, that is, the stars are O-rich which is in stark contrast to the appearance in the optical and infrared spectra of strong bands of C-containing molecules. For the coolest stars $\left(T_{\text {eff }}<5500 \mathrm{~K}\right), \mathrm{C} / \mathrm{He}$ ratios of less than about $0.5 \%$ result in an input $\mathrm{C}$ abundance less than the derived total $\mathrm{O}$ abundance. For the mean $\mathrm{C} / \mathrm{He}$ ratio $(=0.7 \%)$ of the EHes, the stars will be C-rich but barely so.

The $C_{2}$ Phillips system lines $0-2 \mathrm{Q}(34), 1-3 \mathrm{Q}(8)$, and 1-3 $\mathrm{Q}(10)$ provide another opportunity to obtain a $\mathrm{C}$ abundance. For the three $\mathrm{HdC}$ stars with detectable $\mathrm{C}_{2}$ lines, the $\mathrm{C}_{2}$ based abundance is 0.8 (HD 137613), 0.7 (HD 175813), and 1.0 (HD 182040) dex larger than the input abundance. This is "a carbon problem." This particular carbon problem appears to be largely resolved for models with $\mathrm{C} / \mathrm{He}=10 \%$; the input abundance is increased by 1.0 dex over that of the $\mathrm{C} / \mathrm{He}=1 \%$ models but the $\mathrm{C}$ abundance needed to fit the $\mathrm{C}_{2}$ lines is only slightly increased over that from the $\mathrm{C} / \mathrm{He}=1 \%$ models.

Not only is a $\mathrm{C} / \mathrm{He}=10 \%$ ratio at odds with the much lower ratio of the EHes that is supposed to be generally applicable to $\mathrm{HdC}$ stars but the $\mathrm{C}$ abundance from the $\mathrm{C}_{\mathrm{I}}$ lines which matched the input abundance of the $\mathrm{C} / \mathrm{He}=1 \%$ models is only slightly increased by use of the $\mathrm{C} / \mathrm{He}=10 \%$ models and, therefore, removal of the carbon problem posed by the $\mathrm{C}_{2}$ lines is accompanied by the creation of a carbon problem of about 1 dex from the $\mathrm{C}$ I lines.

Here, our emphasis on the ${ }^{16} \mathrm{O} /{ }^{18} \mathrm{O}$ ratio plausibly allows us to postpone a search for the solution to these carbon problems. Isotopic ratios are insensitive to the adopted $\mathrm{C} / \mathrm{He}$ ratio and may not depend greatly on the correct solution to the carbon problems. Certainly, the presence of a very low ${ }^{16} \mathrm{O} /{ }^{18} \mathrm{O}$ will not be denied.

In our analysis, we, as noted above, adopt a model's input $\mathrm{C}$ abundance in extracting the $\mathrm{N}$ and $\mathrm{O}$ abundances from the $\mathrm{CN}$ and $\mathrm{CO}$ lines. Extracted abundances change by less than $0.2 \mathrm{dex}$ when models with $\mathrm{C} / \mathrm{He}$ ratios from $0.1 \%$ to $10 \%$ are used for the analysis. This is certainly a fortunate circumstance. The derived total elemental $\mathrm{C}, \mathrm{N}$, and $\mathrm{O}$ abundances given in Table 2 are for $\mathrm{C} / \mathrm{He}=1 \%$ and the assumed input composition of heavier elements (i.e., $\log \varepsilon(\mathrm{Fe})=7.2, \log \varepsilon(\mathrm{Na})=6.8, \log \varepsilon(\mathrm{Si})=7.7$, etc.; Asplund et al. 1997). The formal error in the total $\mathrm{N}$ and $\mathrm{O}$ abundances taking into account variations of the atmospheric parameters used in the modeling are estimated to be on the order of $0.3-0.4$ dex.

The HdC HD 137613 was analyzed previously by Kipper (2002) with C, N, and O from $\mathrm{C}_{2}$ Swan system, CN Red system, and $\mathrm{O}$ I lines. Our derived abundances for HD 137613 are in poor agreement with those reported by Kipper (2002) from an abundance analysis of the $4780 \AA$ to $6400 \AA$ spectrum. For $(\log \epsilon(\mathrm{C}), \log \epsilon(\mathrm{N}), \log \epsilon(\mathrm{O}))$, our results are $(9.5,9.4,8.7)$ whereas Kipper reported values of $(9.3,8.7,7.6)$. Kipper (2002) noted that his analysis of $\mathrm{C}_{2}$ Swan system bands led to a $\mathrm{C}$ abundance 0.2 dex less than the input abundance, which 
considering the uncertainties in the analysis indicates that the carbon problem is diminished or absent for this HdC. (Kipper, however, considered that the carbon problem was present.) A detailed discussion of the abundance differences between this and Kipper's study is not attempted here where we focus on the isotopic $\mathrm{O}$ ratios.

Asplund et al. (2000) obtained C, N, and O abundances for Y Mus in their analysis of warm RCBs. Their $\mathrm{C}$ abundance exhibits the carbon problem in that the optical C $\mathrm{I}$ lines gave $\log \epsilon(\mathrm{C})=$ 8.9 for models with the input abundance of 9.5. Their $\mathrm{N}$ abundance of 8.8 contrasts with our value of 9.4. Unfortunately, we are unable to determine the $\mathrm{O}$ abundance.

\subsection{Na and $S$ Abundances}

Several atomic lines (Na I, S I, Mg I, Fe I, C I, etc.) are identified in our spectra of the warmer $\mathrm{HdC}$ stars. $\mathrm{Na}$ and $\mathrm{S}$ are the only elements showing two (or more) unblended absorption lines for the five $\mathrm{HdC}$ stars and their abundances will be more reliable. Abundances of $\mathrm{Na}$ and $\mathrm{S}$ in Table 2 are from models with $\mathrm{C} / \mathrm{He}=1 \%$. These values are unchanged if the metallicity (abundance of elements heavier than $\mathrm{O}$ ) is decreased from its quasi-solar value; the electrons contributing to the $\mathrm{He}^{-}$ continuous opacity are donated by $\mathrm{C}$ atoms primarily. Obviously with $\mathrm{He}^{-}$as an opacity source, the derived $\mathrm{Na}$ and $\mathrm{S}$ abundances are dependent on the $\mathrm{C} / \mathrm{He}$ ratio assumed in constructing the model atmosphere: the change from $\mathrm{C} / \mathrm{He}=1 \%$ to $10 \%$ results in about a 0.6 dex increase in the $\mathrm{Na}$ and $\mathrm{S}$ abundances.

Sodium abundances were obtained for the five HdC stars. The region providing the $2.33 \mu \mathrm{m} \mathrm{Na}$ I doublet was not observed for the RCBs. The $\mathrm{Na}$ abundances from the $\mathrm{C} / \mathrm{He}=1 \%$ models run from $\log \epsilon(\mathrm{Na})=6.5$ to 7.0 for a mean abundance of 6.8. The $\mathrm{S}$ abundance has been estimated for all of our stars except HD 173409 for which the region providing the S I lines was not observed. The mean $S$ abundance is $\log \varepsilon(S)=7.5$ for the HdC stars and 6.8 for the RCB stars S Aps and Y Mus. The photospheric spectrum of UW Cen is diluted by circumstellar emission (see above) and, hence, the derived S abundance is an underestimate.

For the $\mathrm{HdCs}$, the $\mathrm{Na}$ and $\mathrm{S}$ abundances are suprasolar from the $\mathrm{C} / \mathrm{He}=1 \%$ models; if we assume $[\mathrm{Na} / \mathrm{Fe}]=[\mathrm{S} / \mathrm{Fe}]=0$, the implied $\mathrm{Fe}$ abundances are +0.2 to +0.5 dex above solar. The $\mathrm{Mg}$ I, Si I, and Fe I generally confirm these Fe overabundances. However, the RCBs S Aps and Y Mus give the subsolar S abundance expected of EHes and RCBs. The Fe abundance for $\mathrm{Y}$ Mus from one $\mathrm{Fe} \mathrm{I}$ line is also subsolar. The $\mathrm{S}$ abundance in Table 2 for Y Mus is within 0.1 dex of the value reported by Asplund et al. (2000) from optical spectra, a difference well within the errors of measurement.

Few direct comparisons with the literature are possible for these $\mathrm{Na}$ and $\mathrm{S}$ abundances. The Na abundance for HD 137613 is 0.9 dex greater than Kipper's (2002) from optical spectra. Kipper did not determine a $\mathrm{S}$ abundance but his abundances for other $\alpha$-elements $(\mathrm{Mg}, \mathrm{Si}, \mathrm{Ca}$, and $\mathrm{Ti})$ are -0.8 dex less than solar, while our $\mathrm{S}$ abundance is slightly suprasolar.

For the $\mathrm{C} / \mathrm{He}=10 \%$ models, the $\mathrm{Fe}$ range for the $\mathrm{HdC}$ stars is lifted to +0.8 to +1.1 above solar-an impossible interval. Models with a $\mathrm{C} / \mathrm{He}$ below $1 \%$ will result in lower $\mathrm{Na}$ and $\mathrm{S}$ abundances but, as noted above, there is a lower limit to the $\mathrm{C} / \mathrm{He}$ ratio not too much less than $1 \%$ below which the $\mathrm{HdC}$ appears oxygen rich. That limit will not suffice to reduce $\mathrm{Na}$ and $\mathrm{S}$ below their solar abundances. In short, the $\mathrm{Na}$ and $\mathrm{S}$ abundances present an interesting problem within the constraints set by the

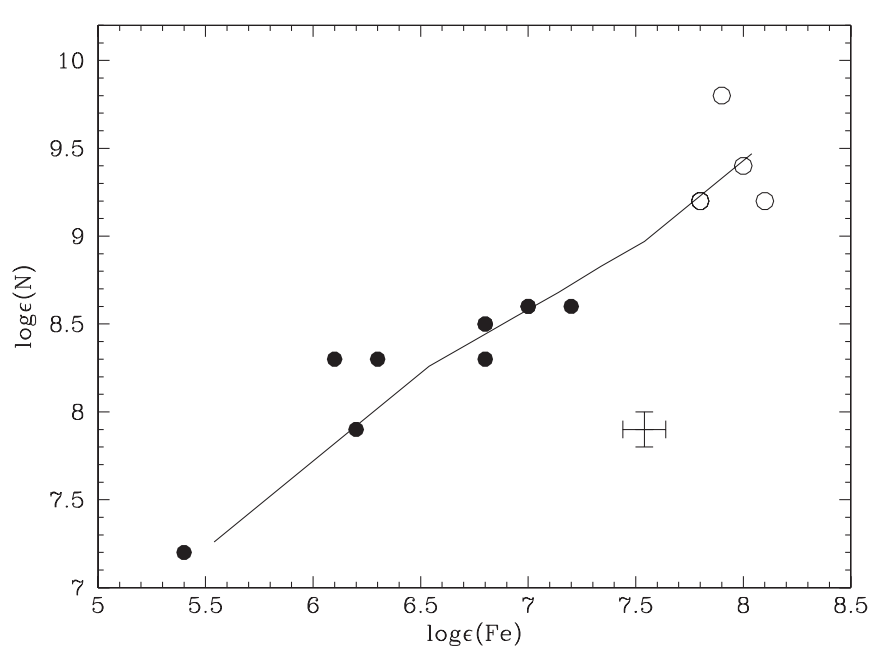

Figure 23. Nitrogen abundances for EHe stars (filled circles) and HdC stars (open circles) as a function of the Fe abundances. The solid line represents the $\mathrm{N}$ abundance being equal to the sum of the initial $\mathrm{C}, \mathrm{N}$, and $\mathrm{O}$ abundances expected from the $\mathrm{Fe}$ abundance. The solar $\mathrm{N}$ and $\mathrm{Fe}$ abundances are indicated by a large cross.

assumption of local thermodynamic equilibrium for the models and the analysis of the lines.

\subsection{Compositions Along the HdC, RCB, and EHe Sequence}

If the working hypothesis that the three classes of $\mathrm{H}$-deficient luminous stars share a common evolutionary scenario, their chemical compositions should also share some common characteristics. Since these stars are supergiants with presumably deep convective envelopes that may achieve dredge-up of nuclear processed material from an He-burning shell, there may be changes of composition along the evolutionary track(s) linking the $\mathrm{HdC}, \mathrm{RCB}$, and EHe stars.

It is evident that EHe stars are not all of the same composition. The metallicity spans over two orders of magnitude with relative abundances of the elements which are unaffected by changes during stellar evolution having the values shown by normal $(\mathrm{H}-$ rich) stars (Pandey et al. 2006). Elements affected by evolution include $\mathrm{He}, \mathrm{C}, \mathrm{N}, \mathrm{O}$ and, in a few cases, the $s$-process elements. The few EHes and RCBs having extraordinarily high $\mathrm{Si} / \mathrm{Fe}$ and $\mathrm{S} / \mathrm{Fe}$ ratios are termed "minority" EHes and RCBs (Lambert $\&$ Rao 1994) and are ignored here. HdC stars all appear to be majority representatives.

The EHe stars are a useful reference because they are immune to the carbon problem affecting the warm RCB stars. Abundance information on EHes is taken from Pandey et al. (2001), Pandey et al. (2006), and Pandey \& Reddy (2006). As shown by Pandey et al. (2006), addition of data drawn from the literature for other EHes would not affect our conclusions. In Figure 23, N abundances for EHe stars are shown as a function of the $\mathrm{Fe}$ abundances. The EHes form a sequence in this figure with, as shown by Pandey et al. (2006), the $\mathrm{N}$ abundance being essentially equal to the sum of the initial $\mathrm{C}, \mathrm{N}$, and $\mathrm{O}$ abundances (the solid line in the figure) expected from the $\mathrm{Fe}$ abundance which is assumed to be unaffected by the ravages of stellar evolution. The interpretation is that the nitrogen is contributed by a layer in which CNO-cycling has converted initial $\mathrm{CNO}$ to $\mathrm{N}$ and that this layer is now the major component of the $\mathrm{HdC}$ atmosphere/envelope. The $\mathrm{HdCs}$ placed in this figure using the $\mathrm{Fe}$ abundance inferred from the mean of their $\mathrm{Na}$ and $\mathrm{S}$ abundances lie on the extension of the EHes sequence; they are apparently remarkably Fe-rich and N-rich. On the assumption 


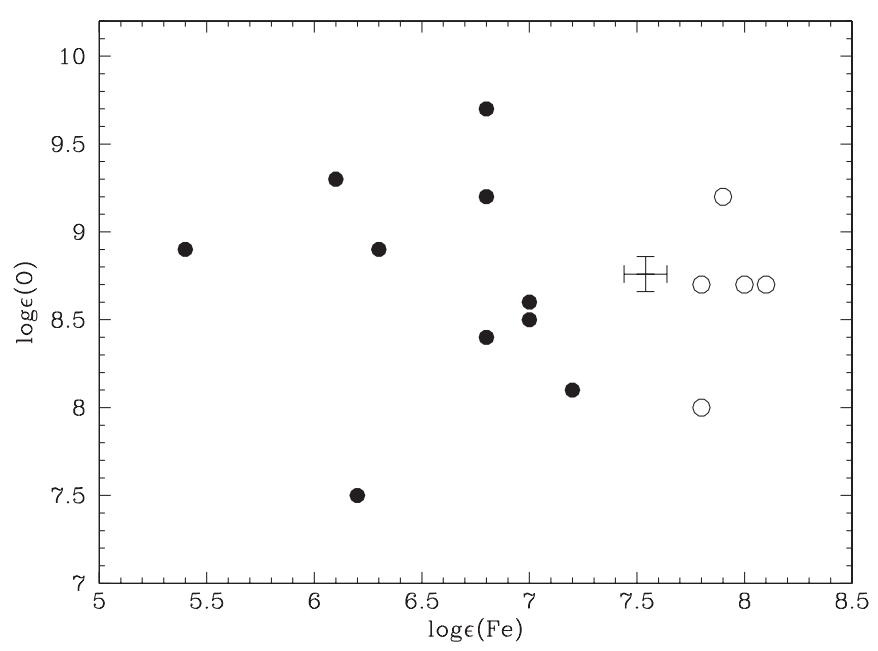

Figure 24. Oxygen abundances for EHe stars (filled circles) and HdC stars (open circles) as a function of the Fe abundances. As in Figure 23, the solar O and $\mathrm{Fe}$ abundances are indicated by a large cross.

that the initial CNO abundances scale linearly with the initial $\mathrm{Fe}$ abundance for suprasolar metal-rich compositions, the HdCs are also consistent with the above idea about the origin of $\mathrm{N}$. The warm RCB stars (not shown in Figure 23) show a similar trend for $\mathrm{N}$ with $\mathrm{Fe}$ with data from Asplund et al. (2000) and Rao \& Lambert $(2002$, 2008). Three minority RCBs anchor the trend at the low Fe end. The RCB N-Fe relation is somewhat offset from the EHe trend: superposition requires either a shift of the RCB $\mathrm{N}$ abundances by about -0.4 dex, or the Fe abundances by +0.4 dex, or a mix of $\mathrm{N}$ and Fe shifts. The shifts may reflect systematic errors associated with the abundance analysis that left the RCB's carbon problem unresolved.

In Figure 24, the $\mathrm{O}$ abundances of EHe stars are shown as a function of the $\mathrm{Fe}$ abundances. The $\mathrm{O}$ abundances show a considerable scatter at all well-sampled values of the $\mathrm{Fe}$ abundance, a scatter not shown by the $\mathrm{N}$ abundances. The range in $\mathrm{O}$ abundances is not dissimilar for the $\mathrm{HdC}$ and EHe stars. The isotopic mix of the $\mathrm{O}$ in EHes is unknown. For three of the $\mathrm{HdC}$ stars, we know that ${ }^{18} \mathrm{O}$ not ${ }^{16} \mathrm{O}$ is the dominant isotope. Anticipating that ${ }^{18} \mathrm{O}$ is synthesized from ${ }^{14} \mathrm{~N}$ (see the next section), it is important to note that the ratio of ${ }^{18} \mathrm{O} / \mathrm{N}$ varies from star to star. This ratio for the four stars with detections of $\mathrm{C}^{18} \mathrm{O}$ ranges from a high of 0.25 to a low of 0.04 .

It is apparent from Figure 24 that the majority of the $\mathrm{O}$ abundances equal or exceed the initial $\mathrm{O}$ abundance predicted by the $\mathrm{Fe}$ abundance and the usual $\mathrm{O}-\mathrm{Fe}$ relation for initial abundances. (The warm RCBs-not shown in Figure 24also show a scatter in the $\mathrm{O}$ versus $\mathrm{Fe}$ diagram and, as for the EHes, the isotopic mix of the $\mathrm{O}$ in unknown. On average, the RCB stars are offset from the EHe stars by about +0.6 dex in O.) The $\mathrm{O}$ abundances of the EHe and HdC stars exceed the low abundances expected for ${ }^{16} \mathrm{O}$ after $\mathrm{CNO}$-cycling has converted initial $\mathrm{CNO}$ to $\mathrm{N}$. Survival of ${ }^{16} \mathrm{O}$ requires a temperature of less than about 15 million degrees. The ${ }^{16} \mathrm{O}$ after CNO-cycle equilibrium is depleted by a factor of about 0.9 dex for cycle operation at 20 million degrees, and depletion increases with increasing temperature. Once $\mathrm{O}$ depletion occurs, the ${ }^{16} \mathrm{O} /{ }^{18} \mathrm{O}$ ratio becomes very high $\left(\sim 10^{4}\right.$ at 20 million degrees) and the ${ }^{17} \mathrm{O}$ abundance exceeds that of ${ }^{18} \mathrm{O}$ (by a factor of $\sim 5$ at 20 million degrees; Arnould et al. 1999). Clearly, the nitrogen and oxygen abundances of the EHe, RCB, and $\mathrm{HdC}$ stars seem unlikely to both be residues from $\mathrm{H}$ - burning via the CNO-cycles. ${ }^{9}$ This conclusion is absolutely certain for the $\mathrm{HdC}$ stars where $\mathrm{O}$ isotopic abundances are available.

\section{SITES FOR THE SYNTHESIS OF ${ }^{18} \mathrm{O}$}

Discovery of a dramatic enrichment of ${ }^{18} \mathrm{O}$ in HD 137613 by Clayton et al. (2005) was anticipated by Warner (1967). Clayton et al.'s (2007) extension of the discovery to two other HdC stars and four RCBs showed that theories of the evolution of these $\mathrm{H}$-deficient stars must provide for the common occurrence of abundant ${ }^{18} \mathrm{O}$. Theory in this discipline has two parts: a mechanism of nucleosynthesis that provides abundant ${ }^{18} \mathrm{O}$ and a recipe for stellar evolution that leads to an $\mathrm{H}$-deficient object rich in ${ }^{18} \mathrm{O}$.

The likely heart of the mechanism of ${ }^{18} \mathrm{O}$ synthesis was identified by Warner (1967): ${ }^{14} \mathrm{~N}$, the dominant ash of $\mathrm{H}$ burning, is converted to ${ }^{18} \mathrm{O}$ by ${ }^{14} \mathrm{~N}(\alpha, \gamma){ }^{18} \mathrm{~F}\left(\beta^{+} \nu\right){ }^{18} \mathrm{O}$ in the run up to He-burning but with further increases in temperature another $\alpha$-capture turns the ${ }^{18} \mathrm{O}$ to ${ }^{22} \mathrm{Ne}$ prior to ignition of $\mathrm{He}$ burning. The ${ }^{18} \mathrm{O}$ yield per initial ${ }^{14} \mathrm{~N}$ is obviously dependent on the physical conditions (temperature, density, and composition) and their temporal variation. If high temperatures persist for a long period, ${ }^{14} \mathrm{~N}$ is converted completely to ${ }^{22} \mathrm{Ne}$ and the ${ }^{18} \mathrm{O}$ abundance after achieving a maximum declines to very low levels.

Although the mechanism of ${ }^{18} \mathrm{O}$ synthesis appears broadly understood, the stellar evolution that leads to triggering of the mechanism cannot claim to be understood yet. In large part, the puzzle concerns the site at which ${ }^{18} \mathrm{O}$ is made from ${ }^{14} \mathrm{~N}$ and the source of that ${ }^{14} \mathrm{~N}$.

Clayton et al. (2005) in their discussion of possible origins for the ${ }^{18} \mathrm{O}$ in HD 137613 proposed that its precursor was a star entering the AGB phase of its life. Such a star after He-core burning has a thin ${ }^{18} \mathrm{O}$-rich layer between the $\mathrm{C}-\mathrm{O}$ core and the H-rich envelope. Then, if severe mass loss is invoked to remove the envelope and expose the ${ }^{18} \mathrm{O}$-rich layer, an HD 137613-like star is produced. This invocation of severe mass loss early on the AGB is not only unsupported theoretically by a possible mechanism but may be supposed to produce $\mathrm{H}$ poor stars with a wide range of ${ }^{18} \mathrm{O}$ abundance in contrast to the indication from Clayton et al. (2007) that severe ${ }^{18} \mathrm{O}$ enrichment seems common among the small sample of HdC stars and some RCBs. Clayton et al. (2007) recognized these weaknesses of their 2005 proposal.

Aside from this identification of $\mathrm{H}$-deficient stars with earlyAGB stars, there are, as noted in the Introduction, two scenarios in play to account for H-poor luminous stars- the FF and DD scenarios. Clayton et al. (2007) note that the FF scenario is not expected to achieve an overproduction of ${ }^{18} \mathrm{O}$ because either the ${ }^{14} \mathrm{~N}$ is burnt to ${ }^{22} \mathrm{Ne}$ and, in cases where an H-rich surviving envelope of the post-AGB star is ingested by the final Heshell flash, ${ }^{18} \mathrm{O}$ is efficiently destroyed by proton capture. This expectation is supported by observations of Sakurai's object (V4334 Sgr), a star identified as a FF product, showing the low ${ }^{12} \mathrm{C} /{ }^{13} \mathrm{C}$ ratio characteristic of H-burning. Asplund et al. (1997) found the low ratio from analysis of $\mathrm{C}_{2}$ Swan bands near $4740 \AA$. Their value was confirmed by Pavlenko et al. (2004) from highresolution spectra of strong first-overtone $\mathrm{CO}$ bands. Although these authors do not comment on the presence of ${ }^{12} \mathrm{C}^{18} \mathrm{O}$ bands,

\footnotetext{
9 Note that the high $(>10){ }^{12} \mathrm{C} /{ }^{13} \mathrm{C}$ ratio present in all $\mathrm{HdC}$ and $\mathrm{RCB}$ stars studied so far also rules out the $\mathrm{H}$-burning products in these stars.
} 
it is evident from their illustrated observed and synthetic spectra that the ${ }^{12} \mathrm{C}^{18} \mathrm{O} 2-0$ band must be very weak and the ${ }^{16} \mathrm{O} /{ }^{18} \mathrm{O}$ ratio must be high, i.e., Sakurai's object differs markedly in this regard from the $\mathrm{HdCs}$ with strong $\mathrm{CO}$ bands, as Clayton et al. (2007) remark on the basis of the high-resolution CO spectrum shown first by Geballe et al. (2002).

Although some H-poor stars are outcomes of the FF scenario with Sakurai's object being the most recent notable discovery, studies of the $\mathrm{C}, \mathrm{N}$, and $\mathrm{O}$ elemental abundances for RCB and EHe stars favor the DD scenario. This partiality for the DD scenario was developed without knowledge of the puzzle offered by ${ }^{18} \mathrm{O}$; the stars in the sample are too warm for $\mathrm{CO}$ molecules to survive in their photospheres. ${ }^{10}$

In the DD scenario, an He white dwarf merges with a $\mathrm{C}-$ $\mathrm{O}$ white dwarf as emission of gravitational radiation causes shrinkage of the binary's orbit. The He white dwarf is disrupted and forms a coating on the $\mathrm{C}-\mathrm{O}$ white dwarf. Conversion of gravitational potential to heat causes this coating to swell to supergiant dimensions. Helium-burning maintains the swelling of the star for a brief period to provide an $\mathrm{H}$-deficient luminous star, an EHe, RCB, or an $\mathrm{HdC}$, before descent to the white dwarf cooling track. There has not yet been a consistent discussion of the evolution, internal nucleosynthesis, and changes in surface composition from the time before the merger through to the descent of the H-poor supergiant on to its white dwarf cooling track-it is a difficult theoretical problem. Thus, "toy models" have to be explored as to whether they might sustain high ${ }^{18} \mathrm{O}$ abundances. Three such models are discussed here.

\subsection{Toy Models}

\subsubsection{Model A}

The simplest of the toy models supposes that the merger is cold (i.e., no nucleosynthesis during the merger), and that the composition of the resultant supergiant is determined solely by mixing the He white dwarf with the surface layers of the $\mathrm{C}-\mathrm{O}$ white dwarf; i.e., the surface composition of the supergiant is unaffected by its deep convective envelope and dredge-up of nuclear processed from within the supergiant.

Saio \& Jeffery (2002) and Pandey et al. (2006) argue that this recipe with certain assumptions about the compositions and masses of the He white dwarf and the perturbed surface layers of the $\mathrm{C}-\mathrm{O}$ white dwarf provides a semi-quantitative explanation for the $\mathrm{CNO}$ abundances of EHe and RCB stars but without, of course, a consideration that the oxygen may not be ${ }^{16} \mathrm{O}$, as assumed in the recipe, but ${ }^{18} \mathrm{O}$. In acceptable recipes, the principal ingredients of the envelope of the merged star are about ten parts from the He white dwarf and one part from the outer He-rich layers of the $\mathrm{C}-\mathrm{O}$ white dwarf. These proportions are set by the observation that the $\mathrm{C} / \mathrm{He} \sim 0.01$ for the $\mathrm{EHe}$ stars and the anticipated $\mathrm{He}$ and $\mathrm{C}$ abundances of the $\mathrm{He}$ and $\mathrm{C}-$ $\mathrm{O}$ white dwarf contributions. Added according to taste may be minor amounts of normal composition material from an H-rich layer atop the He-white dwarf and a small contribution from the outer layers of the $\mathrm{C}-\mathrm{O}$ white dwarf immediately below the star's He-rich layer. The carbon, as ${ }^{12} \mathrm{C}$, in such recipes is provided by either the He-rich skin and/or the $\mathrm{C}-\mathrm{O}$ rich layers of the $\mathrm{C}-\mathrm{O}$ white dwarf below this skin. About $90 \%$ of the

\footnotetext{
10 Tenenbaum et al. (2005) found no correlation between dust formation and $\mathrm{CO}$ band strength. However, there is the intriguing possibility that $\mathrm{CO}$ molecules may be detectable in circumstellar gas at and around the time a $\mathrm{RCB}$ star is in decline. Cold $\mathrm{C}_{2}$ molecules have been detected at this time for two RCBs (Rao \& Lambert 2000, 2008).
}

nitrogen in these recipes is provided by the He white dwarf and about $10 \%$ by the old $\mathrm{He}$-shell of the $\mathrm{C}-\mathrm{O}$ white dwarf with an abundance in both cases, it is assumed, equal to the sum of the initial $\mathrm{C}, \mathrm{N}$, and $\mathrm{O}$ abundances, as a result of $\mathrm{CNO}$-cycling. Thus, the observation that the $\mathrm{N}$ abundance is equal to the sum of the initial $\mathrm{C}, \mathrm{N}$, and $\mathrm{O}$ is reproduced by the model. Oxygen, as ${ }^{16} \mathrm{O}$, in these recipes is contributed by the $\mathrm{C}-\mathrm{O}$ white dwarf from its He-rich layer or the scooped up outer layers of its $\mathrm{C}-\mathrm{O}$ rich core. With plausible ratios for the $\mathrm{C} / \mathrm{O}$ abundances of these layers it is possible to reproduce the $\mathrm{O}$ abundances of the $\mathrm{EHe}$ stars.

Given the number of the free parameters in the recipe, it is not surprising that the observed $\mathrm{He}, \mathrm{C}, \mathrm{N}$, and $\mathrm{O}$ abundances of the EHe stars can be reproduced. Indeed, one might say that it is the observed abundances that restrict the choices of mass ratio of material from the $\mathrm{He}$ and $\mathrm{C}-\mathrm{O}$ white dwarfs, and the ratio of mass fractions of $\mathrm{C}$ to $\mathrm{O}$ in the material contributed by the $\mathrm{C}-\mathrm{O}$ white dwarf. Yet, it is important to ask where in a family of such recipes might ${ }^{18} \mathrm{O}$ be included to provide a star after the cold merger with an abundance comparable to or exceeding that of ${ }^{16} \mathrm{O}$ ?

Two suspects may be arraigned: the He white dwarf and the He-shell of the $\mathrm{C}-\mathrm{O}$ white dwarf. Perhaps, a fraction of the He white dwarf in its evolution before onset of electron degeneracy achieved temperatures sufficient to convert ${ }^{14} \mathrm{~N}$ to ${ }^{18} \mathrm{O}$. The reductions of the ${ }^{14} \mathrm{~N}$ implied by the observed ${ }^{18} \mathrm{O} / \mathrm{N}$ $(<0.25)$ ratios do not within the observational errors negate the proposition that the $\mathrm{N}$ abundance within the observational errors are equal to the sum of the initial $\mathrm{C}, \mathrm{N}$, and $\mathrm{O}$ abundances. Perhaps, the more plausible site for the ${ }^{18} \mathrm{O}$ is the He-shell around the $\mathrm{C}-\mathrm{O}$ white dwarf. In evolution to the white dwarf stage, this layer was a bridge between H-burning on one side and He-burning on the other side and, therefore, a part of the $\mathrm{He}$-shell may have experienced partial conversion of $\mathrm{N}$ to ${ }^{18} \mathrm{O}$. The ${ }^{16} \mathrm{O} /{ }^{18} \mathrm{O}$ ratio then depends on the degree of conversion of $\mathrm{N}$ to ${ }^{18} \mathrm{O}$ in the $\mathrm{He}$-shell and the contributions to ${ }^{16} \mathrm{O}$ from the $\mathrm{He}$-shell and the $\mathrm{C}-\mathrm{O}$ white dwarf.

\subsubsection{Model B}

In this family of models, the merger is not cold but hot; i.e., nucleosynthesis occurs during the merger, and detailed studies of the supergiant's evolution with dredge-up of nuclearprocessed material are neglected. Certainly, all DD models assume that heating by gravitational potential energy and Heburning provides expansion of the envelope and creation (and maintenance) of an H-poor supergiant. Clayton et al. (2007) suggested that capture of the He white dwarf by the $\mathrm{C}-\mathrm{O}$ white dwarf triggers an episode of nucleosynthesis lasting several years. In this episode, the nuclear processes include conversion of ${ }^{14} \mathrm{~N}$ in the accreted material to ${ }^{18} \mathrm{O}$ but destruction of ${ }^{18} \mathrm{O}$ by another $\alpha$-capture is considered inhibited by the cessation of conditions favorable for $\alpha$-capture. With a reaction network activated by a "parametric" representation of the temporal variation of the physical conditions, production of ${ }^{18} \mathrm{O}$ was demonstrated as possible during the merger. Clayton et al.'s (2007) abstract notes that "The merger process is estimated to take only a few days, with accretion rates of $150 M_{\odot} \mathrm{yr}^{-1}$ producing a temperature at the base of the accreted envelope of 1.2-1.9 × $10^{8} \mathrm{~K}$." Nucleosynthesis at the envelope's base continues for about 100 years. Invocation of $\alpha$-capture on ${ }^{14} \mathrm{~N}$ as the source of ${ }^{18} \mathrm{O}$ here, as in other models, requires that nucleosynthesis be cut off before the ${ }^{14} \mathrm{~N}$ and ${ }^{18} \mathrm{O}$ become exhausted. 


\subsubsection{Model C}

The merger of the He white dwarf with the $\mathrm{C}-\mathrm{O}$ white dwarf results in the expansion of the envelope to supergiant dimensions. This supergiant is initially hot (i.e., an EHe or a hotter star) but quickly becomes a yellow supergiant (i.e., RCB or $\mathrm{HdC}$ ). Evolution is terminated by a rapid crossing back to a hot supergiant before descent of the white dwarf cooling track. Changes to the surface composition may occur during life as a supergiant if the convective envelope's base reaches sufficiently deep into the star.

Exploratory calculations of the effects of the dredge-up were undertaken by Saio \& Jeffery (2002). In contrast to the rapid merger rate $\left(150 M_{\odot} \mathrm{yr}^{-1}\right)$ adopted by Clayton et al. (2007), Saio \& Jeffery adopted the much slower rate of $10^{-5} M_{\odot} \mathrm{yr}^{-1}$ : "approximately one-half of the Eddington limit accretion rate for white dwarfs" (Saio \& Jeffery 2002). A Clayton et al. style merger is complete in a few days but the post-merger nucleosynthesis is allowed to continue for about 100 years. In contrast, accretion of (say) a $0.3 M_{\odot}$ He white dwarf takes 30,000 years at the Saio \& Jeffery rate. Since accretion at this rate suffices to drive the transition from a white dwarf to an $\mathrm{H}$-poor supergiant in much less than 30,000 years, the principal phase of accretion as the H-poor supergiant with the embedded merging pair of stars is gaining in mass.

Illustrative calculations shown by Saio \& Jeffery (2002; their Figures 3, 4, and 5) for accretion of $\mathrm{H}$-free material suggest that the supergiant's convective envelope may not closely approach the He-burning shell in the initial evolution from EHe to HdC. Just prior to the final rapid evolution back to higher effective temperatures, a close approach of the base of the convective envelope to the top of the He-burning shell may introduce a difference in composition along the sequence HdC-RCB-EHe. On the basis of these calculations, it would appear that the surface abundances may not differ greatly from those provided by the cold merger (models of type A); i.e., the convective envelope does not dredge up appreciable amounts of material processed within the supergiant. If $\mathrm{H}$ is included in the accreted material, the convective envelope may encompass the H-burning shell in the early phases of life as a cool H-poor supergiant with ensuing changes in surface composition. Abundance of ${ }^{18} \mathrm{O}$ was not reported on by Saio \& Jeffery (2002).

Obviously, additional calculations are needed to determine if and under what conditions ${ }^{18} \mathrm{O}$ may be produced at levels up to ${ }^{18} \mathrm{O} / \mathrm{N} \sim 0.3$. It will be apparent that the ingredients in such calculations are numerous (mass accretion rate, mass and composition of the He white dwarf, mass and composition of the outer layers of the $\mathrm{C}-\mathrm{O}$ white dwarf that are mixed with the accreted material,....). These ingredients define and influence the evolution of the resulting H-poor supergiant and its surface composition including changes resulting from dredge-up by its deep convective envelope. Much remains to be done.

\section{CONCLUDING REMARKS}

On the observational front, the apparent difference in the ${ }^{16} \mathrm{O} /{ }^{18} \mathrm{O}$ ratios of the $\mathrm{HdC}$ and $\mathrm{RCB}$ stars is intriguing. Is this simply an artifact from examining the necessarily small (complete) sample of HdC stars and a small, if incomplete, sample of cool RCB stars? Or is the difference a clue to these stars' origins? In order to shed light on these questions, it will be necessary to find more of HdC stars and to observe more of the known cool RCBs. The total sample of HdC stars has remained at five since the epochal paper by Warner (1967).
On the other hand, valuable additional data can be provided for RCBs. High-resolution spectra of the first-overtone and especially the fundamental $\mathrm{CO}$ bands should provide a more secure estimate of the ${ }^{16} \mathrm{O} /{ }^{18} \mathrm{O}$ ratio than those obtained by Clayton et al. (2007) from medium-resolution spectra. Our result for S Aps from first-overtone CO bands supports this contention.

Published exploratory calculations of the nucleosynthesis achieved during the DD merger and subsequently deserve to be developed. One hopes that valuable predictions will emerge about the range of and correlations between the $\mathrm{C}, \mathrm{N}$, and $\mathrm{O}$ elemental and isotopic abundances and the abundance of other elements affected by the nucleosynthesis, such as $\mathrm{Li}, \mathrm{F}$, and the trans-iron elements produced by the $s$-process. As part of a test of predictions, it would be desirable to address the (different) carbon problems of the RCBs and HdCs.

Synthesis of fluorine occurs as indicated by observations of high F abundances for EHe (Pandey 2006) and RCBs (Pandey et al. 2008). Clayton et al. (2007) discuss F synthesis in their parametric modeling of a hot merger but the maximum $\mathrm{F}$ abundance achieved is below the observed values. A particularly fascinating challenge is offered by the presence of lithium in one HdC star (HD 148839; Rao \& Lambert 1996) and four RCB stars (Asplund et al. 2000). Conditions under which ${ }^{7} \mathrm{Li}$ is produced by the Cameron \& Fowler (1971) mechanism from ${ }^{3} \mathrm{He}$ are incompatible with those for synthesis of ${ }^{18} \mathrm{O}$ from ${ }^{14} \mathrm{~N}$ and $\mathrm{F}$ synthesis. Survival of $\mathrm{Li}$ in regions capable of synthesis of ${ }^{18} \mathrm{O}$ from ${ }^{14} \mathrm{~N}$ is impossible given that $\mathrm{Li}$ is destroyed by $\alpha$-capture orders of magnitude faster than ${ }^{14} \mathrm{~N}$ is converted to ${ }^{18} \mathrm{O}$ via the intermediary ${ }^{18} \mathrm{~F}$. Unfortunately, the Li-rich $\mathrm{RCB}$ stars are among those for which the first-overtone $\mathrm{CO}$ bands are or are expected to be absent or too weak for a determination of the O isotopic ratio. Our analysis of HD 148839 offers the tantalizing result that this Li-rich star may indeed have a lower ${ }^{18} \mathrm{O}$ abundance than other (Li-poor) HdC stars. High-resolution infrared spectra of the $\mathrm{CO}$ fundamental of much higher $\mathrm{S} / \mathrm{N}$ ratio would be of interest.

The FF scenario may result in $\mathrm{Li}$ synthesis but is not expected to account for ${ }^{18} \mathrm{O}$-rich stars. Production of ${ }^{7} \mathrm{Li}$ is predicted (Herwig \& Langer 2001) by the Cameron \& Fowler (1971) mechanism in which ${ }^{3} \mathrm{He}$ is converted by $\alpha$-capture and subsequent electron-capture to ${ }^{7} \mathrm{Li}$. In the DD scenario which can potentially account for the ${ }^{18} \mathrm{O}$-rich stars, neither the $\mathrm{He}$ nor the $\mathrm{C}-\mathrm{O}$ white dwarf can be expected to contain ${ }^{3} \mathrm{He}$, the necessary ingredient for Li synthesis. Clayton et al. (2007) sketched how the nucleosynthesis may be modified by adding an H-rich envelope (presumably a potential reservoir of ${ }^{3} \mathrm{He}$ ) to the He white dwarf. Their sketch did not cover lithium synthesis. As in other areas of stellar astrophysics, lithium is at the center of very fascinating questions of stellar nucleosynthesis and evolution.

This paper is based on observations obtained at the Gemini Observatory, which is operated by the Association of Universities for Research in Astronomy, Inc., under a cooperative agreement with the NSF on behalf of the Gemini partnership: the National Science Foundation (United States), the Science and Technology Facilities Council (United Kingdom), the National Research Council (Canada), CONICYT (Chile), the Australian Research Council (Australia), Ministério da Ciência e Tecnologia (Brazil), and SECYT (Argentina). The observations were obtained with the Phoenix infrared spectrograph, which was developed and is operated by the National Optical Astronomy Observatory. The spectra were obtained as part of programs 
GS-2006A-C-13 and GS-2007A-DD-1. This research has been supported in part by the Robert A. Welch Foundation of Houston, Texas. K.E. gratefully acknowledges support from the Swedish Research Council.

\section{REFERENCES}

Alvarez, R., \& Plez, B. 1998, A\&A, 330, 1109

Arnould, M., Goriely, S., \& Jorissen, A. 1999, A\&A, 347, 572

Asplund, M., Gustafsson, B., Kiselman, D., \& Eriksson, K. 1997, A\&A, 318, 251

Asplund, M., Gustafsson, B., Lambert, D. L., \& Rao, N. K. 2000, A\&A, 353, 287

Asplund, M., Gustafsson, B., Rao, N. K., \& Lambert, D. L. 1998, A\&A, 332, 651

Blöcker, T. 2001, Ap\&SS, 275, 1

Cameron, A. G. W., \& Fowler, W. A. 1971, ApJ, 164, 111

Clayton, G. C., Geballe, T. R., Herwig, F., Fryer, C., \& Asplund, M. 2007, ApJ, 662,1220

Clayton, G. C., Herwig, F., Geballe, T. R., Asplund, M., Tenenbaum, E. D., Engelbracht, C. W., \& Gordon, K. D. 2005, ApJ, 623, L141

Clayton, G. C., Kerber, F., Pirzkal, N., De Marco, O., Crowther, P. A., \& Fedrow, J. M. 2006, ApJ, 646, L69

Costes, M., Naulin, C., \& Dorthe, G. 1990, A\&A, 232, 270

Davis, S. P., Abrams, M. C., Phillips, J. G., \& Rao, M. L. P. 1988, JOSA B, 5, 2280

Davis, S., Wallace, L., Brault, J., \& Engleman, R. 2005, The CN Spectrum from the Infrared to the Ultraviolet (NSO Technical Report; Tucson, AZ: National Solar Observatory)

Decin, L., et al. 2003, A\&A, 400, 679

Fujita, Y., \& Tsuji, T. 1977, PASJ, 29, 711

Geballe, T. R., Evans, A., Smalley, B., Tyne, V. H., \& Eyres, S. P. S. 2002, Ap\&SS, 279, 39

Goorvitch, D. 1994, ApJS, 95, 535

Guerrero, J., García-Berro, E., \& Isern, J. 2004, A\&A, 413, 257

Gustafsson, B., \& Asplund, M. 1996, in ASP Conf. Ser. 96, Hydrogen Deficient Stars, ed. C. S. Jeffery \& U. Heber (San Francisco, CA: ASP), 27

Herwig, F. 2001, Ap\&SS, 275, 15

Herwig, F., \& Langer, N. 2001, MmSAI, 72, 277

Hill, V., et al. 2002, A\&A, 387, 560

Hinkle, K., Wallace, L., \& Livingston, W. C. 1995, Infrared Atlas of the Arcturus Spectrum, 0.9-5.3 microns (San Francisco, CA: ASP)
Hinkle, K. H., et al. 2003, Proc. SPIE, 4834, 353

Huber, K. P., \& Herzberg, G. 1979, Molecular Spectra and Molecular Structure. IV. Constants of Diatomic Molecules (New York: Van Nostrand)

Iben, I. J., \& Tutukov, A. V. 1984, ApJS, 54, 335

Iben, I. J., Tutukov, A. V., \& Yungelson, L. R. 1996, ApJ, 475, 291

Johansson, L. 1966, Ark Fys, 31, 201

Kipper, T. 2002, Balt. Astron., 11, 249

Kokkin, D. L., Bacskay, G. B., \& Schmidt, T. W. 2007, J. Chem. Phys., 126, 084302

Kupka, F., Piskunov, N., Ryabchikova, T. A., Stempels, H. C., \& Weiss, W. W. 1999, A\&AS, 138, 119

Lambert, D. L., Gustafsson, B., Eriksson, K., \& Hinkle, K. H. 1986, ApJS, 62, 373

Lambert, D. L., \& Rao, N. K. 1994, J. Astron.\&Astrophys., 15, 47

Lawson, W. A., Cottrell, P. L., Kilmartin, P. M., \& Gilmore, A. C. 1990, MNRAS, 247,91

Moore, C. E. 1993, Tables of Spectra of Hydrogen, Carbon, Nitrogen, and Oxygen Atoms and Ions (Boca Raton, FL: CRC Press)

Pandey, G. 2006, ApJ, 648, L143

Pandey, G., Lambert, D. L., Jeffery, C. S., \& Rao, N. K. 2006, ApJ, 638, 454

Pandey, G., Lambert, D. L., \& Rao, N. K. Rao 2008, ApJ, 674, 1068

Pandey, G., Rao, N. K., Lambert, D. L., Jeffery, C. S., \& Asplund, M. 2001, MNRAS, 324, 937

Pandey, G., \& Reddy, B. E. 2006, MNRAS, 369, 1677

Pavlenko, Ya. V., Geballe, T. R., Evans, A., Smalley, B., Eyres, S. P. S., Tyne, V. H., \& Yakovina, L. A. 2004, A\&A, 417, L39

Rao, N. K. 2005, in ASP Conf. Ser. 336, Cosmic Abundances as Records of Stellar Evolution and Nucleosynthesis, ed. T. G. Barnes, III \& F. N. Bash. (San Francisco, CA: ASP), 185

Rao, N. K., \& Lambert, D. L. 1996, in ASP Conf. Ser. 96, Hydrogen Deficient Stars, ed. C. S. Jeffery \& U. Heber (San Francisco, CA: ASP), 43

Rao, N. K., \& Lambert, D. L. 2000, MNRAS, 313, L33

Rao, N. K., \& Lambert, D. L. 2002, PASP, 115, 1304

Rao, N. K., \& Lambert, D. L. 2008, MNRAS, 384, 477

Saio, H., \& Jeffery, C. S. 2002, MNRAS, 333, 121

Tenenbaum, E. D., et al. 2005, AJ, 130, 256

Urdahl, R. S., Bao, Y., \& Jackson, W. M. 1991, CPL, 178, 425

Wallace, L., \& Hinkle, K. 2007, ApJS, 169, 159

Wallace, L., \& Livingston, W. C. 2003, An atlas of the Solar Spectrum in the Infrared from 1850 to $9000 \mathrm{~cm}^{-1}$ (1.1 to $5.4 \mu \mathrm{m}$ ) (revised; Tucson, AZ: NOAO)

Warner, B. 1967, MNRAS, 137, 119

Webbink, R. F. 1984, ApJ, 277, 355 